OPEN ACCESS

Edited by:

Mohammad Hosein Farzaei,

Kermanshah University of Medical

Sciences, Iran

Reviewed by:

Wang Ning,

Anhui University of Chinese Medicine,

China

Shi-Jun Yue,

Shaanxi University of Chinese

Medicine, China

Chenglong Xie,

First Affiliated Hospital of Wenzhou

Medical University, China

Chengyao Ma,

Affiliated Hospital of Nanjing University

of Chinese Medicine, China

*Correspondence:

Yu He

heyu0923@sina.com

Specialty section:

This article was submitted to

Ethnopharmacology,

a section of the journal

Frontiers in Pharmacology

Received: 31 March 2021 Accepted: 29 November 2021 Published: 17 January 2022

Citation:

Huang $P$, Wan $\mathrm{H}$, Shao C, Li C,

Zhang $L$ and He $Y$ (2022) Recent Advances in Chinese Herbal Medicine

for Cerebral Ischemic

Reperfusion Injury.

Front. Pharmacol. 12:688596.

doi: 10.3389/fphar.2021.688596

\section{Recent Advances in Chinese Herbal Medicine for Cerebral Ischemic Reperfusion Injury}

\author{
Ping Huang ${ }^{1}$, Haitong $\mathrm{Wan}^{2}$, Chongyu Shao ${ }^{2}$, Chang $\mathrm{Li}^{2}$, Ling Zhang ${ }^{2}$ and $\mathrm{Yu} \mathrm{He}^{1 *}$ \\ ${ }^{1}$ School of Pharmaceutical Sciences, Zhejiang Chinese Medical University, Hangzhou, China, ${ }^{2}$ School of Life Sciences, Zhejiang \\ Chinese Medical University, Hangzhou, China
}

Cerebral ischemic reperfusion injury $(\mathrm{Cl} / \mathrm{Rl})$ is a critical factor that leads to a poor prognosis in patients with ischemic stroke. It is an extremely complicated pathological process that is clinically characterized by high rates of disability and mortality. Current available treatments for $\mathrm{Cl} / \mathrm{RI}$, including mechanical and drug therapies, are often accompanied by significant side effects. Therefore, it is necessary to discovery new strategies for treating $\mathrm{Cl} / \mathrm{RI}$. Many studies confirm that Chinese herbal medicine (CHM) was used as a potential drug for treatment of $\mathrm{Cl} /$ $\mathrm{RI}$ with the advantages of abundant resources, good efficacy, and few side effects. In this paper, we investigate the latest drug discoveries and advancements on $\mathrm{Cl} / \mathrm{Rl}$, make an overview of relevant $\mathrm{CHM}$, and systematically summarize the pathophysiology of $\mathrm{Cl} / \mathrm{RI}$. In addition, the protective effect and mechanism of related $\mathrm{CHM}$, which includes extraction of single $\mathrm{CHM}$ and $\mathrm{CHM}$ formulation and preparation, are discussed. Moreover, an outline of the limitations of $\mathrm{CHM}$ and the challenges we faced are also presented. This review will be helpful for researchers further propelling the advancement of drugs and supplying more knowledge to support the application of previous discoveries in clinical drug applications against $\mathrm{Cl} / \mathrm{RI}$.

Keywords: Chinese herbal medicine, cerebral ischemia, reperfusion injury, pathophysiology, pharmacological effects, mechanisms

\section{INTRODUCTION}

Cerebral ischemia (CI), a universal cerebrovascular disease with intracranial ischemia and hypoxia, often occurs in stroke or "brain attack" and results in local tissue necrosis and secondary apoptosis. Ischemic stroke accounts for $85 \%$ of all strokes, and hemorrhagic stroke accounts for $15 \%$ (Rai et al., 2016). It is characterized by sudden onset, high risk of disease, high mortality rate, and long-term disability, which brings a severe burden to the normal life of individuals and the cost of the medical care system (Liu R. et al., 2020). With about 15 million cases occurring every year (Manwani and McCullough, 2013) and a 30\% mortality rate, CI is regarded as the third leading cause of death and a major cause of disability worldwide (Wang R. et al., 2019). The patients of CI should be diagnosed rapidly and treated promptly and appropriately; otherwise, it is very difficult to cure.

Saving the damaged neurons and recovering the impaired neuronal function are two main measures for treating ischemic stroke clinically. Most ischemic stroke survivors suffer irreversible neurological damage that worsens their quality of life. Currently, magnetic resonance imaging and cranial computed tomography (CT) are used only for diagnosis, whereas patients need effective drug intervention at once to achieve a good prognosis. Up to now, intravenous thrombolysis with Alteplase (rt-PA) is the only FDA-approved treatment for ischemic stroke, which carries a restrictive therapeutic window within $4.5 \mathrm{~h}$ to achieve the treatment of ischemic stroke (Catanese et al., 2017). 


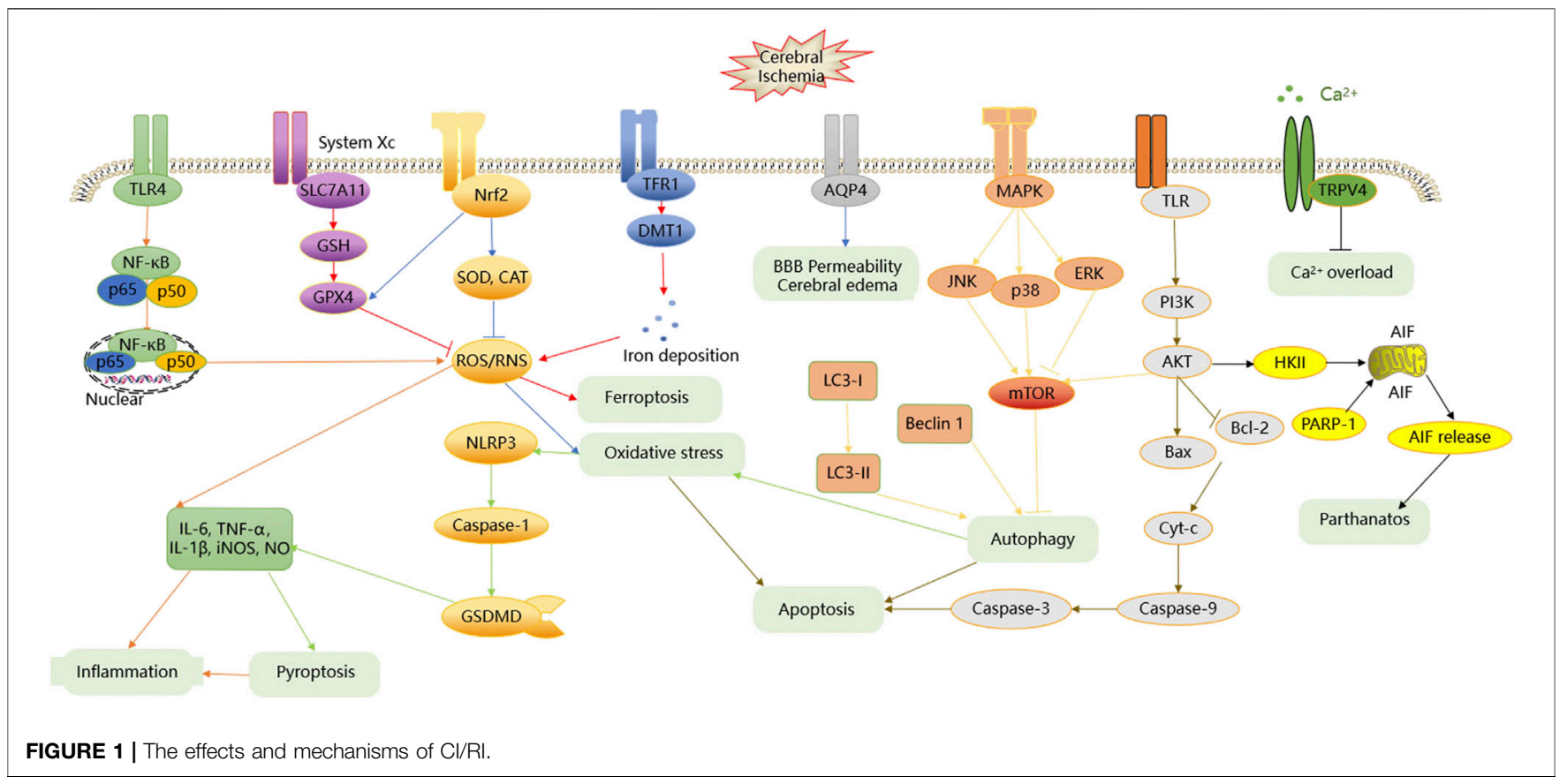

There is no virtual progress in the treatment of CI from only a limited number of available drugs in recent years. Once CI occurs, restoration of blood oxygen supply in time is the first principle of current clinical treatment, and the patients suffer the injury and cascade reactions caused by $\mathrm{CI}$ and reperfusion. CI reperfusion injury (CI/RI), a complicated pathological process, usually happens in succession with CI. Therefore, it is necessary to develop new drugs or therapies to lengthen the therapeutic window and ameliorate the consequences of CI/RI.

The pathological mechanism of $\mathrm{CI} / \mathrm{RI}$ is extremely complex and involves many factors, such as energy metabolism disorders, $\mathrm{Ca}^{2+}$ overload, inflammatory and oxidative stress, excitotoxicity, ferroptosis, apoptosis (Auriel and Bornstein, 2010), and some novel forms of programmed cell death. Autophagy, ferroptosis, parthanatos, and pyroptosis, as the novel forms of programmed cell death, have attracted much attention in recent years. During reperfusion, CI is only partially salvaged, but further damages flood in. The procedure of injury and remedy in the brain may interlace together, which makes it extremely difficult to develop effective treatments for $\mathrm{CI} / \mathrm{RI}$ with such complex factors and their interactions. In conclusion, the prevention of $\mathrm{CI} / \mathrm{RI}$ can decrease its incidence and mortality.

Chinese herbal medicine (CHM) maintains an irreplaceable position in the health system all the time in China. Recently, it has also been recognized as a fertile source of novel lead molecules for modern drug discovery in other countries (Long et al., 2012). With the increasing trend of CI/RI, more and more researchers have fixed their eyes on exploring CHM, which has the characteristics of multi-ingredient, multitarget, and multipathway to work together. CHM functions holistically and shows an obvious superiority over "one drug, one target" treatment, especially in treating multifactorial diseases, such as $\mathrm{CI} / \mathrm{RI}$. Routinely, CHM has many therapeutic effects for $\mathrm{CI} / \mathrm{RI}$, such as antioxidation; anti-inflammation; antiapoptosis; promotion of angiogenesis; enhancement of autophagy; adjustment of energy metabolism; protection of blood-brain barrier (BBB) integrity; prevention of $\mathrm{Ca}^{2+}$ overload; and regulation of the neurological cell proliferation, differentiation, and regeneration. The latest studies are making progress in borneol intervention ( $\mathrm{Li}$ et al., 2021), polysaccharide intervention (Meng et al., 2021) for CI/RI, and reviewing phytochemicals as regulators of microglia/ macrophage activation in CI/RI (Subedi and Gaire, 2021). Li et al. and Meng et al. comprehensively highlight the potential application of borneol and polysaccharides as a neuroprotective agent against CI/RI. Subedi et al. makes significant progress in the study of phytochemicals and their derivatives as promising drug candidates for the treatment of tissue inflammatory injury. This review article focuses on the role of extraction components of single $\mathrm{CHM}$, CHM formulas, and CHM preparations that have protective effects in animal and cell CI/RI models by multiple mechanisms. To explore the complex mechanism of $\mathrm{CHM}$ against $\mathrm{CI} / \mathrm{RI}$, we summarize the effects of the CHM used to treat CI/RI and their associated mechanisms. Figure 1 summarizes the etiopathogenesis of $\mathrm{CI} / \mathrm{RI}$, and the tables represent CHM's probable effects and mechanism of neuroprotection activity in CI/RI models. This work opens a new window for drug discovery and brings a better understanding of the therapeutic principles of $\mathrm{CHM}$ for CI/RI. It is valuable for further promoting candidate drug development and providing more citation-based information that can be applied in clinical drug application against CI/RI.

\section{SURVEY METHODOLOGY}

"PubMed," "ScienceDirect," and "CNKI" databases are mainly used to search for published articles using the search terms 
"traditional Chinese medicines," "Chinese herbal medicine," "natural medicine," "natural drug," "crude drugs," "cerebral," "brain," "ischemia," and "stroke" in the "title/abstract" field. Related articles were chosen manually before December 31, 2020. Both animal experiments (in vivo) and cell studies (in vitro) on $\mathrm{CHM}$ intervention in $\mathrm{CI} / \mathrm{RI}$ are included in the review.

\section{ETIOPATHOGENESIS OF CI/RI}

According to the clinical study, CI/RI arises due to blockage in the blood vessels caused by thrombus, hemorrhage in the brain. $\mathrm{CI} /$ $\mathrm{RI}$ is often induced by complicated pathologic factors, such as inflammation, oxidative stress, apoptosis, $\mathrm{Ca}^{2+}$ overload, autophagy, and many others. The major signaling pathways in $\mathrm{CI} / \mathrm{RI}$ include PI3K/AKT, MAPKs, NF- $\kappa \mathrm{B}, \mathrm{Nrf} 2$, and others. Moreover, the numerous abovementioned factors or signaling pathways that lead to CI/RI are related to and induced with each other, eventually resulting in apoptosis or nerve necrosis in the ischemic region. The detailed process is summarized in Figure 1.

\subsection{Inflammation}

Inflammation response is a key element conducive to the pathogenic process (Dong et al., 2019; Lambertsen et al., 2019) and involved in all stages (Boehncke, 2018; O'Connell et al., 2017) of $\mathrm{CI} / \mathrm{RI}$, which is triggered by toll-like receptor 4 (TLR4) activation. TLR4, a member of the TLRs family, activates a typical transcription factor nuclear factor-kappa B (NF- $\mathrm{B})$. When cells are irritated by extracellular matters, viral products or bacterial components through TLR4 and the NF- $\kappa B$ nuclear localization site are exposed. Free NF- $\kappa \mathrm{B}$ migrates to the nucleus at a high rate of speed, binds to specific $\kappa \mathrm{B}$ sequences, and then transcribes in the nucleus (P65 or P50) after combining with promoters and enhancers of specific gene sequences (SantaCecilia et al., 2016; Wu et al., 2020). Once activating NF-кB p65 or NF- $\kappa$ B p50, inflammatory cells, such as lymphocytes, leukocytes, neutrophils, and macrophages, actively infiltrate into the brain, and then overproduced reactive oxygen species (ROS) are released. ROS promotes glial activation and neutrophil infiltration and contributes to the release of pro-inflammatory cytokines, such as tumor necrosis factor- $\alpha$ (TNF- $\alpha$ ), interleukin$1 \beta$ (IL-1 $\beta$ ), IL-6, inducible nitric oxide synthase (iNOS), and nitric oxide (NO) (Kim et al., 2014; Anrather and Iadecola, 2016). After a while, some pro-inflammatory cytokines, such as TNF- $\alpha$, IL-1 $\beta$, and NO, exacerbate and block microvessels, further inducing the release of cytokines by activating leukocytes, and result in secondary neuronal damage and inflammation exacerbation (Ji et al., 2016; Moritz et al., 2017). Transforming growth factor- $\beta 1$ (TGF- $\beta 1$ ), IL-4, and IL-10, as antiinflammatory cytokines, suppress the secretion of proinflammatory cytokines (Kim et al., 2014). If the level of antiinflammatory cytokines is higher than the pro-inflammatory cytokine expression, the inflammation response is mitigated.

\subsection{Oxidative Stress}

Oxidative stress easily happens during CI/RI because of the more oxygen consuming; the overproduction of ROS, reactive nitrogen species (RNS), and polyunsaturated fatty acids; the fewer contents of endogenous antioxidants, and the weaker antifree radical system (Du et al., 2012). It often results from the imbalance of ROS/RNS production and antioxidant factors of cells (Kopani et al., 2006). Under normal physiological conditions, ROS can be scavenged by endogenous antioxidant enzymes, such as superoxide dismutase (SOD), catalase (CAT), and glutathione peroxidase (GPX), which are proven to combat oxidative stress (Son et al., 2010; Li et al., 2013). Nuclear factor erythroid 2-related factor (Nrf2), a pleiotropic transcription factor and the key genomic homeostatic regulator, can regulate the expression of endogenous antioxidant enzymes (Wu Q. Q. et al., 2011). However, superfluous production of ROS/RNS causes energy impairment, lipid peroxidation, mitochondrial dysfunction, accumulation of aggregated proteins, and damage of intracellular biofilm lipids, leading to oxidative stress (Olmez and Ozyurt, 2012; Navarro-Yepes et al., 2014). Oxidative stress not only leads to systemic oxidative damage, but also triggers apoptosis, pyroptosis, and inflammation.

\subsection{Apoptosis}

Apoptosis, one of the primary patterns of neuronal death, plays a critical role in pathogenesis and prognosis. TLRs activate downstream adaptor signaling molecules such as phosphatidylinositol-3-kinase-serine/threonine kinase (PI3K/ AKT). Phosphorylation of PI3K/AKT can affect the B-cell leukemia/lymphoma 2 (Bcl-2) protein family, which includes the antiapoptotic protein $\mathrm{Bcl}-2$. There is a proapoptotic protein called $\mathrm{Bcl}$ 2-associated $\mathrm{X}$ (Bax), which blocks programmed cell death. The Bcl-2 protein family mediates apoptosis by opening the mitochondrial permeability transition pore, thereupon bringing the release of cytochrome c (Cyt-c) from the mitochondria intermembrane space to the cytoplasm (Vela et al., 2013). Apoptosis is also caused by the activation of cysteine-aspartic proteases (Caspases), which hydrolyzes and cleaves relevant proteins and regulates cell death. In all the Caspases, Caspase-9, and Caspase-3 expression are often found in the CI rat model (Geng et al., 2013). Caspase-3, the effector/executioner compound, the cleavage of which leads to DNA fragmentation, degradation, and cross-linking of related proteins, then induces cell apoptosis directly. Caspase-9, the initiator molecule formed with Cyt-c, activates Caspase-3 and gives rise to irreversible cell death (Chen et al., 2015).

\section{4 $\mathrm{Ca}^{2+}$ Overload}

$\mathrm{Ca}^{2+}$ overload is supposed to be one of the first events induced by $\mathrm{CI} / \mathrm{RI}$. During CI/RI, the content of adenosine triphosphate (ATP) sharply drops, the cellular function is beyond paired due to energy loss, and subsequent injury in the brain is hard to restore. Under these circumstances, abundant extracellular $\mathrm{Ca}^{2+}$ outflow through $\mathrm{Ca}^{2+}$ channels into cells is called " $\mathrm{Ca}^{2+}$ overload" or " $\mathrm{Ca}^{2+}$ toxicity." Overloaded $\mathrm{Ca}^{2+}$ combines with death signals to facilitate procedure change of cellular components and death by apoptosis or necrosis or straightly acts on catabolic enzymes that cause cell demise (Orrenius et al., 2003). 
Transient receptor potential vanilloid 4 (TRPV4), a member of the TRP family, is widely distributed in neurons, smooth muscle cells, and glial cells. The activation of the TRPV4 channel accelerates the opening of intermediate conductance $\mathrm{Kca}$ $\left(\mathrm{IK}_{\mathrm{Ca}}\right)$, small conductance $\mathrm{Kca}\left(\mathrm{SK}_{\mathrm{Ca}}\right)$, and large conductance $\mathrm{Ca}^{2+}$-activated $\mathrm{K}^{+}\left(\mathrm{BK}_{\mathrm{Ca}}\right)$ channels and reduces the accumulation of a potentially pathological level of $\mathrm{Ca}^{2+}$ from a number of potential sources of influx to prevent $\mathrm{Ca}^{2+}$ overload (Sonkusare et al., 2012; Szarka et al., 2018). The intracellular $\mathrm{Ca}^{2+}$ overload brings out all kinds of $\mathrm{Ca}^{2+}$-dependent physiological reactions, such as the disintegration of phospholipids, proteins, and nucleic acids and the disruption of the structure and function of the cell membrane, accelerating cell death finally (Berliocchi et al., 2005).

\subsection{Autophagy}

Autophagy, a stylized cell survival process, has caught many researchers' eyes as a novel effect of CI/RI (Hou et al., 2019). Moderate autophagy maintains cell metabolism and homeostasis to protect nerve cells (Wang $\mathrm{P}$ et al., 2018). If the autophagy is activated excessively, it leads to cell death and aggravation of CI/ RI. The cellular events that occur during autophagy follow distinct stages: autophagy initiation, autophagosomal membrane nucleation, the fusion of the autophagosome with the lysosome (Wang M et al., 2018). The mitogen-activated protein kinase (MAPK) and PI3K/AKT pathway are responsible for the induction of autophagy. The MAPK family is composed of c-Jun N-terminal protein kinases (JNK), p38, and extracellular signal-regulated kinases (ERK). The phosphorylation of JNK and p38, the activation PI3K/AKT signal pathway, and the suppression of ERK, can motivate the mTOR signaling and then lead to autophagy initiation (Kyriakis and Avruch 2012). Beclin 1 takes part in the nucleation of the autophagosomal membrane and becomes the marker of final autophagosome formation. The microtubule-associated protein 1 light chain 3 (LC3) is the first kind of protein identified from the autophagosome membrane. During the autophagy process, LC3-I is hydrolyzed into LC3-II. Therefore, the expression of beclin 1 or LC3-II/LC3-I ratio is considered as the major criterion for judging the autophagy level (Mizushima, 2010; Mizushima and Komatsu, 2011). The final stage is the fusion of autophagosome and lysosome. The autophagy process may be conducive to neural death in a way. There is a report showing that inhibition of autophagy can block the cathepsins tBid-mitochondrial apoptotic signaling pathway via stabilization of the lysosomal membrane in ischemic astrocytes in the model of middle cerebral artery occlusion (MCAO) rats and oxygen glucose deprivation (OGD) cells (Zhou X. Y. et al., 2017).

\subsection{Ferroptosis}

Ferroptosis, a newly programmed type of regulated cell death, is the focus and hot spot of research on the treatment and prognosis improvement of many diseases in recent years (Lei et al., 2019; Qiu et al., 2020). The two key features of the ferroptosis process are the accumulation of lipid peroxidates and the metabolism of iron ions. The solute carrier family 7A11 (SLC7A11), a subunit of system Xc-, is a part of an important antioxidant system in cells. The downregulation of SLC7A11 causes suppression of glutathione $(\mathrm{GSH})$ biosynthesis and the subsequent inhibition of GPX4 activity, which leads to the accumulation of lipid ROS and the activation of ferroptosis (Li J. et al., 2020). Furthermore, the deposition of iron brings ferroptosis through producing ROS by the Fenton reaction. The transferrin receptor 1 (TFR1) transports the ferric iron $\left(\mathrm{Fe}^{3+}\right)$ into the endosome, and then $\mathrm{Fe}^{3+}$ converses into the ferrous iron $\left(\mathrm{Fe}^{2+}\right)$. After that, the divalent metal transporter 1 (DMT1) mediates $\mathrm{Fe}^{2+}$ to release from the endosome and then stores it in the unstable iron pool and ferritin (Xie et al., 2016). The ferroportin oxidizes $\mathrm{Fe}^{2+}$ to $\mathrm{Fe}^{3+}$, thereby strictly controlling iron homeostasis and inhibiting ferroptosis in cells. It follows that TFR1 and DMT1 are vital proteins to regulate intracellular iron transportation and maintain iron metabolism and homeostasis.

\subsection{Parthanatos}

Parthanatos, a new type of caspase-independent pathophysiology, is found to play an important role during CI/RI lately (Zhang R. et al., 2015; Wang et al., 2016). In parthanatos, mitochondria homeostasis is considered to be the crucial element of cell death (Dawson and Dawson, 2017). The hexokinase-II (HK-II), the isoform of HK, has the N-terminal hydrophobic segment, which binds to mitochondria and is activated by the PI3K/AKT pathway. The poly ADP-ribose polymerase-1 (PARP-1) near the DNA damage site has the ability to repair DNA. The activation of PARP1 and HK-II trigger mitochondrial membrane depolarization and induce apoptosis inducing factor (AIF) released from mitochondria to the nucleus, which then results in DNA fragmentation, dysfunction of mitochondria, and collapse of cells (Wang et al., 2011; Nederlof et al., 2014; Robinson et al., 2019).

\subsection{Pyroptosis}

Pyroptosis, a novel and special form of programmed cell death, brings an inflammation response and aggravates damage (Zeng et al., 2019). Activating the NOD-like receptor protein 3 (NLRP3) inflammasome, which controls maturation and secretion of proinflammatory cytokines, is regarded as a crucial pathway in the initiation of pyroptosis (Liu Z. et al., 2017). NLRP3 is activated by an external stimulus, such as oxidative stress, and triggers the Caspase-1 cascade (Barrington et al., 2017). Caspase-1, an essential precursor of pyroptosis, cleaves gasdermin D (GSDMD) into an N-terminal product (GSDMD-NT) and C-terminal fragment (GSDMD-CT) (Shi et al., 2015; Kovacs and Miao, 2017). GSDMD-NT binds to the components of the lipid bilayer, including phosphatidic acid, phosphatidylinositol, and phosphatidylserine, leading to pore generation to execute cell swelling and rupture (Zeng et al., 2019). GSDMD-CT is different from GSDMD-NT; it suppresses the GSDMD-NT when the cell is in a quiescent condition to remain GSDMD in an inactivated status. On the other side, the Caspase-1processed pro-inflammatory cytokines, such as IL-1 $\beta$, IL- 6 , and IL18 , are released through membrane pores formed by GSDMD-NT. This cascade leads to pyroptosis, amplifies the inflammation and then aggravates the brain injury.

\subsection{Other Etiologies}

CI/RI triggers a complex cascade of pathophysiological events. Except for the etiologies reviewed above, there are a great many 
pathological mechanisms that participate in the process of CI/RI, such as BBB permeability, cerebral edema, and angiogenesis. Aquaporin 4 (AQP4), the abundant and important aquaporin, regulates Connexin $43(\mathrm{Cx} 43)$ expression. Matrix metalloprotein 9 (MMP-9), a member of the family of zinc-dependent proteolytic enzymes, degrades almost all the extracellular matrix and basement membrane components. The expression of AQP4 and MMP-9 could severely affect the permeability of the BBB and the degree of cerebral edema (Yao et al., 2015; Maugeri et al., 2016). The tight junction (TJ), a layered cell structure construct, plays a crucial role in the integrity of the BBB. The expression of TJ-associated proteins, such as claudin-5, occluding, and zona occludens-1 (ZO-1), is closely related to BBB permeability (Yang and Rosenberg, 2011). Angiogenesis, including the cell proliferation, migration, and differentiation process, is regulated by many factors such as angiogenin (Ang), vascular endothelial growth factor (VEGF), hypoxia inducible factor (HIF), and transforming growth factor (TGF). Ang-2 facilitates angiogenesis, whereas Ang-1 accelerates the subsistence of endothelial cells and the maturation of blood vessels (Anisimov et al., 2013). TGF- $\beta 1$ induces VEGF to offer a favorable condition for angiogenesis through adjusting the expression of multifarious angiogenic molecules. HIF-1 increases levels of several angiogenic genes to promote forming new blood vessels (Pereira et al., 2014).

\section{CHM FOR TREATING CI}

\subsection{An Overview of Relevant CHM}

$\mathrm{CHM}$ is derived from natural sources and was used clinically in human history. Now, many studies suggest that CHM has many positive effects on the brain, and these effects are beneficial to the treatment and prevention of CI/RI. CHM plays an essential role in the discovery and development of new drug entities, for example, the remarkable contribution of artemisinin in combating malaria, which is threatening millions of lives in developing regions. $\mathrm{CHM}$ includes effective extraction components of single CHM and CHM formulas and preparations. Researchers find that some CHM extractions, such as salvianolic acid B (Jiang et al., 2015; Wang G. et al., 2019), salvianolic acid A (Wang et al., 2012), tanshinone $\mathrm{II}_{\mathrm{A}}$ (Cai et al., 2017; Xu, 2019), ginsenoside Rg1 (Zhang et al., 2013; He et al., 2014; Sun et al., 2014), ginsenoside Rb1 (Zhou Y. et al., 2014; Chen et al., 2015), ginsenoside F1 (Zhang J. et al., 2019), baicalein (Li W. H. et al., 2020), baicalin (Zhou et al., 2016; Zhou Z. Q. et al., 2017), gastrodin (Peng et al., 2015; Liu et al., 2016; Shi et al., 2018), hydroxy-safflor yellow A (Tan et al., 2020), astragaloside IV (Li et al., 2019), astragaloside VI (Chen X et al., 2019), puerarin (Zhou F. et al., 2014; Liu Y. et al., 2017), salidroside (Han et al., 2015), magnolol (Liu X. et al., 2017), tetrahydroxystilbene glucoside (Yu et al., 2019), galangin (Guan et al., 2021), daucosterol palmitate (Zhang $H$. et al., 2020), senkyunolide-H (Zhang J. Y. et al., 2019), paeoniflorin (Guo et al., 2012; Chen et al., 2013), musk ketone (Zhou et al., 2020), procyanidins (Yang et al., 2020), ilexonin A (Zheng et al., 2015; Xu et al., 2016), picroside II (Zhang et al., 2017), geniposide (Li et al., 2016), resveratrol (Sun et al., 2019; Zhang, 2019), hispidulin (An et al., 2019), and (+)-borneol (Chang et al., 2017), have a potent neuroprotection effect against CI/RI. Here, Figure 2 represents chemical structures related to compounds from single CHM that improves CI/RI. As we all know, in clinical treatment, the application of CHM formulas/preparations is widespread, and most of them are confirmed to have a good therapeutic effect against $\mathrm{CI} / \mathrm{RI}$. CHM protects $\mathrm{CI} / \mathrm{RI}$ from many aspects; it ameliorates oxidative stress, inflammation, and neuron apoptosis; modulates intracellular $\mathrm{Ca}^{2+}$ concentration, ferroptosis, and parthanatos; promotes angiogenesis; and regulates autophagy and pyroptosis. The related intracellular signaling pathways are also intricate. In a word, exploring the mechanism and effective molecular targets of $\mathrm{CHM}$ are important and will provide new insights into the treatment of $\mathrm{CI} / \mathrm{RI}$ and promote the process of discovering active drugs from CHM.

Traditional Chinese medicine theory believes that blood stasis is the fundamental cause of CI/RI. CHM plays an irreplaceable role and provides unique advantages in the management of CI/RI. Some scholars identify that CHM has actions of activating Qi and blood, clearing heat and detoxification, nourishing Yin and generating body fluid, and calming liver wind in treating and preventing CI (Wang, 2016). Increasing evidence indicates CHM might be effective in the treatment of CI/RI, especially CHM with the effects of replenishing and activating blood. Extraction components of single CHM (Table 1), CHM formulas (Table 2), and CHM preparations (Table 3) all show some benefit in removing blood stasis, which drives further studies for ischemic diseases more widely.

\subsection{Extraction Components of Single CHM for Treating $\mathrm{Cl} / \mathrm{RI}$}

\subsubsection{Salvia miltiorrhiza Bunge [Lamiaceae; Salviae miltiorrhizae radix et rhizoma].}

Salvia miltiorrhiza Bunge [Lamiaceae; Salviae miltiorrhizae radix et rhizoma] is one of the typical blood-activating and stasisresolving medicines, and it offers therapeutic promise for cerebrovascular diseases (Ma Y. et al., 2020). Salvianolic acids and tanshinones are the main compounds in Salvia miltiorrhiza Bunge [Lamiaceae; Salviae miltiorrhizae radix et rhizoma]. Salvianolic acid A (SAA) is proved to display potent cerebroprotection through inhibiting soluble epoxide hydrolase $(\mathrm{sEH})$ to increase epoxyeicosatrienoic acid (EET) levels (Wang et al., 2012). Some studies find that salvianolic acid B (SAB) increased the activities of SOD, CAT, and GSH-Px; decreased the content of MDA and the activities of LDH and NOS; improved Bcl-2 expression; and reduced Bax expression to exert the neuroprotective effect in animal models with CI/RI (Jiang et al., 2015; Wang G. et al., 2019). A study conducted in the model of CI/RI mice by $\mathrm{Xu}$ (2019) suggests that tanshinone $\mathrm{II}_{\mathrm{A}}$ inhibits ferroptosis through regulating iron homeostasis, reducing the intracellular ROS level and iron deposition. Another trial identifies that tanshinone $\mathrm{II}_{\mathrm{A}}$ has an antioxidant effect against $\mathrm{CI} / \mathrm{RI}$ in rats. Tanshinone $\mathrm{II}_{\mathrm{A}}$ upregulated the expression of Nrf2 mRNA and the content of Nrf2 protein in 


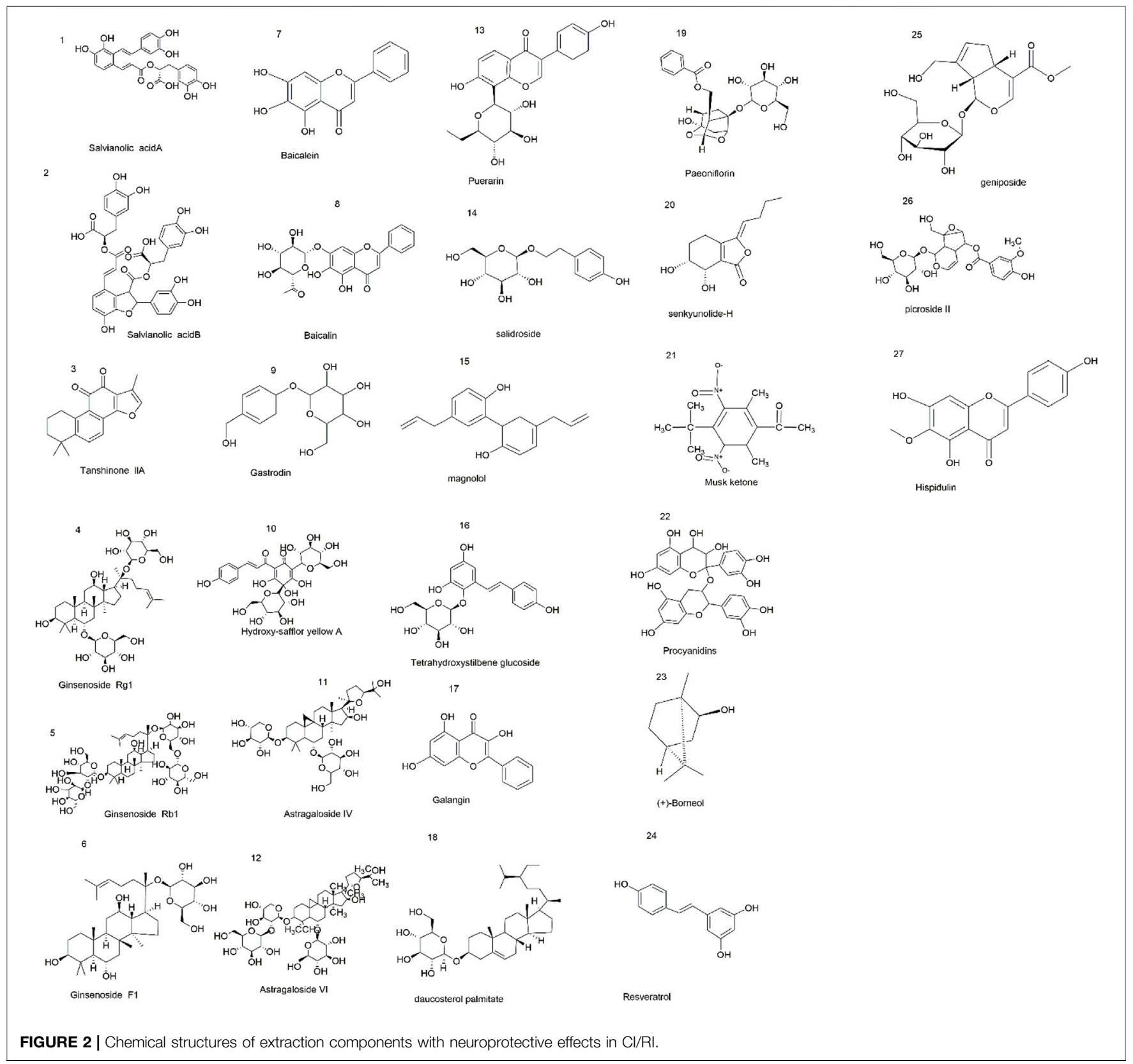

nuclear and then increased the content of antioxidant enzymes and reduced the generation of oxidative productions (Cai et al., 2017).

\subsubsection{Panax ginseng C.A.Mey(Araliaceae; Ginseng Radix et Rhizoma)}

Panax ginseng C.A.Mey(Araliaceae; Ginseng radix et rhizoma), which belongs to the Araliaceae family, has the function of strengthening vital Qi for brain protection. Ginsenosides are considered the main bioactive constituent of ginseng, and some studies report their neuroprotective effects (Ong et al., 2015). Recent research indicates that ginsenoside Rg1 (GRg1) prevents neurotoxicity by blocking $\mathrm{Ca}^{2+}$ over-influx into neuronal cells to elevate intracellular $\mathrm{Ca}^{2+}$ concentration in both MCAO/R-induced rats and OGD/R-induced neuronal cells (Zhang et al., 2013; He et al., 2014; Sun et al., 2014). Ginsenoside Rb1 (GRb1) exerts significant neuroprotective effects on CI/IR both in vivo and in vitro, and these network actions and underlying mechanisms are mediated by antioxidant, anti-inflammatory, and antiapoptotic activities and involve the inhibition of excitotoxicity and $\mathrm{Ca}^{2+}$ influx, preservation of $\mathrm{BBB}$ integrity, and maintenance of energy metabolism (Xie et al., 2021). Ginsenoside F1 (GF1) promotes angiogenesis in vitro and in vivo by activating the insulin-like growth factor 1 /insulin-like growth factor 1 receptor (IGF-1/IGF1R) signaling pathway (whose axis is 
TABLE 1 | Recent advances in protective mechanisms of extraction components of single $\mathrm{CHM}$ for $\mathrm{Cl} / \mathrm{RI}$.

\begin{tabular}{|c|c|c|c|c|c|}
\hline $\begin{array}{l}\text { Single botanical } \\
\text { drugs (main } \\
\text { active ingredients) }\end{array}$ & Object & Effect/Mechanism & Controls & $\begin{array}{c}\text { Minimal active } \\
\text { dosage }\end{array}$ & References \\
\hline $\begin{array}{l}\text { Salvia miltiorrhiza Bunge } \\
\text { [Lamiaceae; Salviae miltiorrhizae } \\
\text { radix et rhizoma] (SAA) }\end{array}$ & MCAO/R-induced rats (in vivo) & $\begin{array}{l}\text { inhibits soluble epoxide } \\
\text { hydrolase (sEH) to increase } \\
\text { epoxyeicosatrienoic acids } \\
\text { (EETs) levels }\end{array}$ & $\begin{array}{l}\text { sham-operated group } \\
\text { (negative control); 14,15- } \\
\text { EEZE group (positive } \\
\text { control) }\end{array}$ & 0.3 mg/kg (i.v.) & $\begin{array}{l}\text { Wang et al. } \\
\text { (2012) }\end{array}$ \\
\hline $\begin{array}{l}\text { Salvia miltiorrhiza Bunge } \\
\text { [Lamiaceae; Salviae miltiorrhizae } \\
\text { radix et rhizoma] (SAB) }\end{array}$ & $\begin{array}{l}\text { MCAO/R-induced mice/rats } \\
\text { (in vivo) }\end{array}$ & $\begin{array}{l}\text { increases the activities of SOD, } \\
\text { CAT, GSH-Px and Bcl-2, } \\
\text { decreases the content of MDA, } \\
\text { LDH, NOS and Bax }\end{array}$ & $\begin{array}{l}\text { sham-operated group } \\
\text { (negative control); } \\
\text { nimodipine group (positive } \\
\text { control) }\end{array}$ & 12 mg/kg (i.p.) & $\begin{array}{l}\text { Jiang et al. } \\
\text { (2015); Wang G. } \\
\text { et al. (2019) }\end{array}$ \\
\hline $\begin{array}{l}\text { Salvia miltiorrhiza Bunge } \\
\text { [Lamiaceae; Salviae miltiorrhizae } \\
\text { radix et rhizoma] (tanshinone IIA) }\end{array}$ & $\begin{array}{l}\text { OGD/R-induced HT22 cells } \\
\text { (in vitro); MCAO/R-induced } \\
\text { mice (in vivo) }\end{array}$ & $\begin{array}{l}\text { inhibits ferroptosis by regulating } \\
\text { the iron homeostasis, reducing } \\
\text { the intracellular ROS level and } \\
\text { the iron deposition; induces }\end{array}$ & $\begin{array}{l}\text { erastin group (negative } \\
\text { control); Nrf2 knockout } \\
\text { mice group (negative } \\
\text { control) }\end{array}$ & $\begin{array}{l}25 \mathrm{mg} / \mathrm{kg} \text { (i.p.); } \\
1 \mu \mathrm{mol} / \mathrm{L} \\
\text { (incubate) }\end{array}$ & $\begin{array}{l}\text { Xu (2019); Cai } \\
\text { et al. (2017) }\end{array}$ \\
\hline
\end{tabular}

Panax ginseng C.A.Mey. (Araliaceae; Ginseng radix et rhizoma) (GRg1)

Salvia miltiorrhiza Bunge [Lamiaceae; Salviae miltiorrhizae radix et rhizoma] (GRb1)

Panax ginseng

C.A.Mey.(Araliaceae; Ginseng radix et rhizoma) (GF1)

Scutellaria baicalensis Georgi [Lamiaceae; Scutellariae radix] (baicalein)

Scutellaria baicalensis Georgi [Lamiaceae; Scutellariae radix] (baicalin)

Gastrodia elata Blume [Orchidaceae; Gastrodiae rhizome] (GAS)

Carthamus tinctorius L. (CTL) [Asteraceae; Carthami Flos] (HSYA)

MCAO/R-induced rats (in vivo); $\mathrm{H}_{2} \mathrm{O}_{2} / \mathrm{R}$-induced cells (in vitro)

MCAO/R-induced rats (in vivo); OGD/R-induced neuronal cells/SH-SY5Y cells (in vitro)

MCAO/R-induced mice/rats (in vivo); OGD/R-induced neuronal cells (in vitro)

2-VO/MCAO/R-induced mice/rats (in vivo); OGD/ R-induced neuronal cells (in vitro)

OGD/R-induced umbilical vein endothelial cells (UVECs); brain microvascular (in vitro) MCAO/R-induced rats (in vivo)

HIE-induced rats (in vivo); OGD/R-induced SH-SY5Y cells (in vitro)

MCAO/R-induced rats (in vivo); $\mathrm{H}_{2} \mathrm{O}_{2} / \mathrm{R}$-induced cells (in vitro)

Astragalus mongholicus Bunge [Fabaceae; Astragali radix] (AIV) oxidation by upregulating the expression of Nrf2

prevents neurotoxicity by

blocking $\mathrm{Ca}^{2+}$ over-influx into

neuronal cells to elevate intracellular $\mathrm{Ca}^{2+}$ concentration

Protects $\mathrm{Cl} / \mathrm{IR}$ by antioxidant, anti-inflammatory, and antiapoptotic activities and involve the inhibition of excitotoxicity and $\mathrm{Ca}^{2+}$ influx preservation of blood-brain barrier (BBB) integrity, and maintenance of energy metabolism

promotes angiogenesis by IGF1/IGF1R signaling pathway

attenuates parthanatos by inhibiting the activation of PARP 1 and diminishing the release of AlF

suppresses apoptosis by regulating NF-kB and PI3K/Akt signaling pathways

improves antioxidant, antiinflammation and antiapoptotic activities by activating Akt/Nrf2 pathway; suppressing expression of Caspase-3, Bax, TNF- $\alpha, I L-1 \beta$, and MDA; upregulating amount of SOD, HO1, SOD1, and Bcl-2 content inhibits pyroptosis by regulating NF-кB signaling pathway and the levels of NLRP3 and Caspase-1; improves antiapoptotic by activating PI3K/Akt signaling pathway

reduces apoptosis and parthanatos by activating AKT to promote HK-II binding to mitochondria; improves antioxidant and antiapoptosis by regulating JAK2/STAT3 signaling pathway
VEGF group (positive control) (nroup (negative control); acetazolamide group (positive control) sham-operated group (negative control); nimodipine group (positive control); LY294002 group (positive control)

\section{$20 \mu \mathrm{mol} / \mathrm{L}$ (incubate) \\ Zhang $\mathrm{J}$ et al., \\ 20 mg/kg (i.p.); $\quad$ He et al. (2014); $5 \mu \mathrm{mol} / \mathrm{L}$ Zhang et al. (incubate) \\ $5 \mathrm{mg} / \mathrm{kg}$ (i.p.); (2013); Sun et al. (2014) $1 \mu \mathrm{mol} / \mathrm{L}$ (incubate) Xie et al. (2021)}

sham-operated group

(negative control)

sham-operated group

(negative control); LY294002 group (positive control) sham-operated group (negative control); nimodipine group (positive control)

sham-operated group (negative control); lexiscan group (positive control)

sham-operated group (negative control); triciribine group and AG490 group (positive control)

\section{0 mg/kg (i.p.) Li W. H et al., 2020}

$1 \mu \mathrm{mol} / \mathrm{L}$ (incubate);

Zhou Z. Q et al. (2017); Zhou 120 mg/kg (i.p.) et al. (2016)

50 mg/kg (i.p.); $\quad$ Peng et al. $15 \mu \mathrm{g} / \mathrm{ml}$ (incubate) (2016); Shi et al. (2018)

10 mg/kg (i.v.); Tan et al. (2020) $10 \mu \mathrm{g} / \mathrm{ml}$ (incubate) (2015); Liu et al. $\begin{array}{ll}15 \mathrm{mg} / \mathrm{kg} \text { (i.v.); } & \text { Li et al. (2019); Xu } \\ 10 \mu \mathrm{g} / \mathrm{ml} & \text { et al. (2020) } \\ \text { (incubate) } & \end{array}$

(Continued on following page) 
TABLE 1 | (Continued) Recent advances in protective mechanisms of extraction components of single $\mathrm{CHM}$ for $\mathrm{Cl} / \mathrm{RI}$.

\begin{tabular}{|c|c|c|c|c|c|}
\hline $\begin{array}{l}\text { Single botanical } \\
\text { drugs (main } \\
\text { active ingredients) }\end{array}$ & Object & Effect/Mechanism & Controls & $\begin{array}{c}\text { Minimal active } \\
\text { dosage }\end{array}$ & References \\
\hline $\begin{array}{l}\text { Astragalus mongholicus Bunge } \\
\text { [Fabaceae; Astragali radix] (AVI) }\end{array}$ & $\begin{array}{l}\text { MCAO/R-induced rats (in } \\
\text { vivo); cultured neural stem } \\
\text { cells (in vitro) }\end{array}$ & $\begin{array}{l}\text { promotes the proliferation and } \\
\text { neurogenesis of cells, and } \\
\text { repairs neurological function of } \\
\text { rats by activating EGFR/MAPK }\end{array}$ & $\begin{array}{l}\text { sham-operated group } \\
\text { (negative control); } \\
\text { gefitinib/PD98059 group } \\
\text { (negative control) }\end{array}$ & $\begin{array}{l}2 \mu \mathrm{g} / \mathrm{kg} \text { (i.v.); } \\
10 \mu \mathrm{g} / \mathrm{ml} \\
\text { (incubate) }\end{array}$ & $\begin{array}{l}\text { Chen X et al., } \\
2019\end{array}$ \\
\hline
\end{tabular}

Pueraria montana var. lobata (Willd.) Maesen and S.M.Almeida ex Sanjappa \& Predeep [Fabaceae; Puerariae lobatae radix] (puerarin)

Rhodiola crenulata (Hook.f. and Thomson) H.Ohba

[Crassulaceae; Rhodiolae

crenulatae radix et

rhizome](salidroside)

Magnolia officinalis Rehder and

E.H.Wilson [Magnoliaceae;

Magnoliae officinalis cortex]

(magnolol)

Reynoutria multiflora (Thunb.)

Moldenke [Polygonaceae;

Polygoni multiflora radix]

(tetrahydroxystilbene glucoside)

Alpinia officinarum Hance

[Zingiberaceae; Alpiniae

officinarum rhizoma] (galangin)

Alpinia oxyphylla Miq.

[Zingiberaceae; Alpiniae

oxyphyllae fructus] (daucosterol

palmitate)

Conioselinum anthriscoides

'Chuanxiong' [Apiaceae;

Chuanxiong rhizoma]

(senkyunolide-H)

Paeonia lactiflora Pall.

[Paeoniaceae; Paeoniae radix

rubra] (paeoniflorin)

Cucumis melo L. [Cucurbitaceae; Trichosanthis fructus] (musk ketone)

grape seeds (procyanidins)

Ilex pubescens Hook. and Arn. [Aquifoliaceae; Ilex pubescens radix] (ilexonin $A$ )

Picrorhiza kurroa Royle ex Benth. [Plantaginaceae; Picrorhizae rhizoma] (picroside II)
MCAO/R-induced rats (in vivo); OGD/R-induced brain vascular endothelial cells (BVECs) (in vitro)

MCAO/R-induced rats (in vivo) signaling pathway

reduces infarct size and brain water content by regulating TLR4/NF- $\mathrm{BB}$ signaling pathway; reduces apoptosis by regulating ERK and PI3K/AKT/mTOR signaling pathways reduces oxidative stress by regulating Nrf2 signaling pathway

MCAO/R-induced mice (in vivo); OGD/R-injured BMECs (in vitro)

MCAO/R-induced mice (in vivo)

suppresses inflammation by inhibiting TNF- $\alpha, I L-1 \beta$, and NO expressions; improves BBB function by increasing $\mathrm{ZO}-1$ and occludin levels

mitigates apoptosis and autophagy by elevating the expressions of NOX4, caspase3/-9, Beclin 1, and the LC3BII// ratio

MCAO/R-induced gerbils (in vivo)

$\mathrm{MCAO} / \mathrm{R}$-induced rats (in vivo) inhibits apoptosis by regulating

nhibits ferroptosis by enhancing the expressions of SLC7A11 and GPX4 $\mathrm{PI}$ KK/Akt/mTOR signaling pathway

MCAO/R-induced mice (in vivo); OGD/R-PC12 cells (in vitro)

MCAO/R-induced mice/rats (in vivo); PDGF-stimulated VSMC/A7r5 cells (in vitro)

MCAO/R-induced rats (in vivo); OGD/R-induced Neura stem cells (NSCs) (in vitro) $\mathrm{MCAO} / \mathrm{R}$-induced rat (in vivo); OGD/R-BV2 cells (in vitro)

MCAO/R-induced rats (in vivo); OGD/R- Neural stem cells (NSCs) (in vitro) inhibits inflammation and apoptosis by activating PI3K/ Akt/NF- $k B$ signaling pathway

ameliorates $\mathrm{Cl}$ and arterial intimal hyperplasia by modulating Ras/MEK/ERK signaling pathway; reduces inflammation by inhibiting MAPKs/NF- $\kappa$ B signaling pathway induces NSC proliferation and differentiation by activating the PI3K/Akt signaling pathway exerts neuroprotective effect by inhibiting TLR4-NLRP3 inflammasome signaling pathway

promotes revascularization, neuronal regeneration, and regulates astrocyte and microglia cell activation; promotes neuronal proliferation and regeneration by regulating Wnt signaling pathway inhibits apoptosis and inflammation by scavenging ROS content and regulating Cyt-c expression

\section{sham-operated group} (negative control)
sham-operated group (negative control)

sham-operated group (negative control); edaravone group (positive control)

sham-operated group (negative control)

sham-operated group (negative control)

sham-operated group (negative control); wortmannin group (positive control) sham-operated group (negative control)

sham-operated group (negative control)

sham-operated group (negative control)

sham-operated group (negative control)

sham-operated group (negative control)
100 mg/kg (i.p.); Zhou F et al.

$10 \mu \mathrm{g} / \mathrm{ml} \quad$ (2014); Liu Z et al. (incubate) (2017)

15 mg/kg (i.p.) Han et al. (2015)

$1.4 \mu \mathrm{g} / \mathrm{kg}$ (i.v.)

Liu X et al., 2017 $1 \mu \mathrm{mol} / \mathrm{L}$

(incubate)

$3 \mathrm{mg} / \mathrm{kg}$ (i.v.)

Yu et al. (2019) 25 mg/kg (i.p.)

Guan et al. (2021)

20 mg/kg (i.p.) Zhang $\mathrm{H}$ et al., 2020

20 mg/kg (i.g.) Zhang J. Y et al., 2019

$0.5 \mathrm{mg} / \mathrm{kg}$ (i.g.); Chen et al. 5 mg/kg (i.p.); (2013); Guo et al. $5 \mu \mathrm{mol} / \mathrm{L}$ (2012) (incubate)

0.5 mg/kg (i.p.) Zhou et al. (2020) 20 mg/kg (i.g.) Yang et al. (2020) 20 mg/kg (i.p.) Xu et al. (2016); Zheng et al. (2015)

sham-operated group (negative control); CyclosporinA group (positive control)

\section{$20 \mathrm{mg} / \mathrm{kg}$ (i.p.) Zhang et al. (2017)}

(Continued on following page) 
TABLE 1 | (Continued) Recent advances in protective mechanisms of extraction components of single $\mathrm{CHM} \mathrm{for} \mathrm{Cl} / \mathrm{RI}$.

\begin{tabular}{|c|c|c|c|c|c|}
\hline $\begin{array}{l}\text { Single botanical } \\
\text { drugs (main } \\
\text { active ingredients) }\end{array}$ & Object & Effect/Mechanism & Controls & $\begin{array}{c}\text { Minimal active } \\
\text { dosage }\end{array}$ & References \\
\hline $\begin{array}{l}\text { Gardenia jasminoides J.Ellis } \\
\text { [Rubiaceae; Gardeniae fructus] } \\
\text { (geniposide) }\end{array}$ & $\begin{array}{l}\text { OGD/R-induced BMECs } \\
\text { (in vitro) }\end{array}$ & $\begin{array}{l}\text { attenuates inflammation by } \\
\text { suppressing ERK1/2 signaling } \\
\text { pathway }\end{array}$ & $\begin{array}{l}\text { sham-operated group } \\
\text { (negative control) }\end{array}$ & $\begin{array}{l}33.2 \mu \mathrm{g} / \mathrm{ml} \\
\text { (incubate) }\end{array}$ & Li et al. (2016) \\
\hline $\begin{array}{l}\text { Polygonum cuspidatum Sieb. et } \\
\text { Zucc. [Polygonaceae; Polygoni } \\
\text { cuspidate radix et rhizoma] } \\
\text { (resveratrol) }\end{array}$ & $\begin{array}{l}\text { MCAO/R-induced rats (in } \\
\text { vivo); } \mathrm{H}_{2} \mathrm{O}_{2} / \mathrm{R} \text {-induced } \mathrm{RSC} 96 \\
\text { cells (in vitro) }\end{array}$ & $\begin{array}{l}\text { inhibits pyroptosis and } \\
\text { autophagy by blocking the } \\
\text { activation of NLRP3 } \\
\text { inflammasome, Caspase-1, } \\
\text { mTOR phosphorylation }\end{array}$ & $\begin{array}{l}\text { sham-operated group } \\
\text { (negative control) }\end{array}$ & $\begin{array}{l}0.1 \mathrm{mmol} / \mathrm{L} \\
\text { (incubate) }\end{array}$ & $\begin{array}{l}\text { Sun et al. (2019); } \\
\text { Zhang (2019) }\end{array}$ \\
\hline $\begin{array}{l}\text { Saussurea involucrata (Kar. and } \\
\text { Kir.) Sch.Bip. [Asteraceae; } \\
\text { Saussureae involucratae flos] } \\
\text { (hispidulin) }\end{array}$ & $\begin{array}{l}\text { MCAO/R-induced rats (in } \\
\text { vivo); OGD/R-induced } \\
\text { astrocytes (in vitro) }\end{array}$ & $\begin{array}{l}\text { suppresses NLRP3-mediated } \\
\text { pyroptosis by modulating the } \\
\text { AMPK/GSK3 } \beta \text { signaling } \\
\text { pathway }\end{array}$ & $\begin{array}{l}\text { sham-operated group } \\
\text { (negative control) }\end{array}$ & $\begin{array}{l}40 \mathrm{mg} / \mathrm{kg} \text { (i.p); } \\
5 \mu \mathrm{mol} / \mathrm{L} \\
\text { (incubate) }\end{array}$ & An et al. (2019) \\
\hline $\begin{array}{l}\text { Blumea balsamifera (L.) DC. } \\
\text { [Asteraceae; Blumi balsamiferae } \\
\text { herba] ((+)-borneol) }\end{array}$ & $\begin{array}{l}\text { MCAO/R-induced rats (in } \\
\text { vivo); lipopolysaccharide } \\
\text { (LPS)-induced BV2 microglial } \\
\text { cells (in vitro) }\end{array}$ & $\begin{array}{l}\text { suppresses inflammation by } \\
\text { reducing production of } \\
\text { proinflammatory cytokines } \\
\text { (iNOS and TNF-a) }\end{array}$ & $\begin{array}{l}\text { sham-operated group } \\
\text { (negative control) }\end{array}$ & 0.5 mg/kg (i.v.) & $\begin{array}{l}\text { Chang et al. } \\
(2017)\end{array}$ \\
\hline $\begin{array}{l}\text { Ginkgo biloba L. [Ginkgoaceae; } \\
\text { Ginkgo folium] (ginkgo diterpene } \\
\text { lactones) }\end{array}$ & $\begin{array}{l}\text { MCAO/R-induced rats (in } \\
\text { vivo); OGD/R-induced cortical } \\
\text { astrocytes (in vitro) }\end{array}$ & $\begin{array}{l}\text { suppresses inflammation by } \\
\text { regulating TLR4/NF-kB } \\
\text { signaling pathway }\end{array}$ & $\begin{array}{l}\text { sham-operated group } \\
\text { (negative control) }\end{array}$ & $\begin{array}{l}1.25 \mathrm{mg} / \mathrm{kg} \text { (i.v.); } \\
12.5 \mu \mathrm{g} / \mathrm{ml} \\
\text { (incubate) }\end{array}$ & LiX. et al., 2020 \\
\hline $\begin{array}{l}\text { Dendrobium nobile Lindl. } \\
\text { [Orchidaceae; Dendrobii caulis] } \\
\text { (dendrobium alkaloids) }\end{array}$ & $\begin{array}{l}\text { MCAO/R-induced rats (in } \\
\text { vivo); OGD/R-induced HT22 } \\
\text { cells (in vitro) }\end{array}$ & $\begin{array}{l}\text { inhibits pyroptosis by reducing } \\
\text { the levels of Caspase- } 1 \text {, } \\
\text { gasdermin-D and inflammatory } \\
\text { factors (IL-1 } 1 \beta \text {, IL-6, and IL-18) }\end{array}$ & $\begin{array}{l}\text { sham-operated group } \\
\text { (negative control); } \\
\text { belnacasan group } \\
\text { (positive control) }\end{array}$ & $\begin{array}{l}0.5 \mathrm{mg} / \mathrm{kg} \text { (i.p.); } \\
0.03 \mathrm{mg} / \mathrm{ml} \\
\text { (incubate) }\end{array}$ & Liu D. et al., 2020 \\
\hline $\begin{array}{l}\text { Ilex pubescens Hook. and Arn. } \\
\text { [Aquifoliaceae; llex pubescens } \\
\text { radix] total flavonoids }\end{array}$ & MCAO/R-induced rats (in vivo) & $\begin{array}{l}\text { suppresses inflammation by } \\
\text { decreasing proinflammatory } \\
\text { cytokines (NO, IL-1 } \beta, T N F-\alpha \text {, } \\
\text { TNOS, iNOS, and cNOS) and } \\
\text { increasing anti-inflammatory } \\
\text { cytokine (IL-10); suppresses } \\
\text { oxidative stress by decreasing } \\
\text { (MDA) level and increasing SOD } \\
\text { content }\end{array}$ & $\begin{array}{l}\text { sham-operated group } \\
\text { (negative control) }\end{array}$ & 100 mg/kg (i.g.) & $\begin{array}{l}\text { Fang et al. (2017); } \\
\text { Miao et al. (2016) }\end{array}$ \\
\hline $\begin{array}{l}\text { Achyranthes bidentata Blume } \\
\text { [Amaranthaceae; Achyranthis } \\
\text { bidentatae radix] (Achyranthes } \\
\text { bidentata polypeptide k) }\end{array}$ & $\begin{array}{l}\text { MCAO/R-induced rats (in } \\
\text { vivo); OGD/R-induced rat } \\
\text { endothelial cells (in vitro) }\end{array}$ & $\begin{array}{l}\text { improves recognition abilities } \\
\text { and neurological outcomes by } \\
\text { modulating NF- } \mathrm{kB} \text { and MMP- } \\
2 /-9\end{array}$ & $\begin{array}{l}\text { sham-operated group } \\
\text { (negative control) }\end{array}$ & $\begin{array}{l}0.1 \mathrm{mg} / \mathrm{kg} \text { (i.v.); } \\
0.2 \mu \mathrm{g} / \mathrm{ml} \\
\text { (incubate) }\end{array}$ & $\begin{array}{l}\text { Cheng et al. } \\
(2019)\end{array}$ \\
\hline $\begin{array}{l}\text { Rhododendron tomentosum } \\
\text { Harmaja [Ericaceae; } \\
\text { Rhododendri flos] total favonoids }\end{array}$ & MCAO/R-induced rats (in vivo) & $\begin{array}{l}\text { reduces } \mathrm{Ca}^{2+} \text { concentration in } \\
\text { the cell by activating BKCa } \\
\text { channel through a TRPV4- } \\
\text { dependent signaling pathway }\end{array}$ & $\begin{array}{l}\text { sham-operated group } \\
\text { (negative control) }\end{array}$ & 100 mg/kg (i.v.) & Han et al. (2018) \\
\hline $\begin{array}{l}\text { Cordyceps sinensis (Berk. Sacc.) } \\
\text { [Clavicipitaceae; Cordyceps] } \\
\text { extract }\end{array}$ & $\begin{array}{l}\text { MCAO/R-induced rats (in } \\
\text { vivo); OGD/R-induced } \\
\text { BMECs (in vitro) }\end{array}$ & $\begin{array}{l}\text { inhibits apoptosis by regulating } \\
\text { the expressions of Bax/Bcl-2, } \\
\text { Cyt-c, and caspase-3/-8/-9 }\end{array}$ & $\begin{array}{l}\text { sham-operated group } \\
\text { (negative control) }\end{array}$ & $\begin{array}{l}1 \mathrm{~g} / \mathrm{kg} \text { (i.g.); } \\
5 \mu \mathrm{g} / \mathrm{ml} \\
\text { (incubate) }\end{array}$ & Bai et al. (2020) \\
\hline $\begin{array}{l}\text { Pinellia pedatisecta Schott } \\
\text { [Araceae; Pinellia rhizoma] } \\
\text { extract }\end{array}$ & $\begin{array}{l}\text { MCAO/R-induced rats (in } \\
\text { vivo); OGD/R-induced UVECs } \\
\text { (in vitro); OGD/R-induced } \\
\text { BMECs (in vitro) }\end{array}$ & $\begin{array}{l}\text { inhibits apoptosis by keeping } \\
\text { the balance between } \mathrm{Bcl}-2 \\
\text { and Bax }\end{array}$ & $\begin{array}{l}\text { sham-operated group } \\
\text { (negative control); } \\
\text { nimodipine group (positive } \\
\text { control) }\end{array}$ & 5 mg/kg (i.g.) & Ye et al. (2016) \\
\hline
\end{tabular}

crucial for cerebral angiogenesis and neurogenesis) in endothelial cells. Meanwhile, it also increased the cerebral microvessel density, improved cerebral blood flow in ischemic regions, and reduced CI/RI, indicating that GF1 is a promising drug for promoting recovery from CI/RI by inducing angiogenesis (Zhang J. et al., 2019).

\subsubsection{Scutellaria baicalensis Georgi [Lamiaceae; Scutellariae Radix]}

Scutellaria baicalensis Georgi [Lamiaceae; Scutellariae radix] is an important CHM with the functions of clearing away heat and dampness, purging fire, and detoxification (Zhao et al., 2019). Baicalein and baicalin are the main active components that are extracted from Scutellaria baicalensis Georgi [Lamiaceae; Scutellariae radix]. Li et al. find that baicalein treatment significantly inhibits the activation of PARP-1 and nuclear translocation of AIF, diminishes the release of AIF in CI/RI rats, and thereby attenuates parthanatos and protects the cerebral tissues from ischemic injury (Li W. H. et al., 2020). Several experiments confirm that baicalin has neuroprotective effects against $\mathrm{CI} / \mathrm{RI}$ by regulating the NF- $\mathrm{KB}$ signaling pathway and $\mathrm{PI} 3 \mathrm{~K} /$ Akt signaling pathway in vitro and in vivo (Zhou Z. Q. et al., 2016; Zhou Z. Q. et al., 2017). 
TABLE 2 | Recent advances in protective mechanisms of $\mathrm{CHM}$ formulations for $\mathrm{Cl} / \mathrm{RI}$.

\begin{tabular}{|c|c|c|c|c|c|}
\hline $\begin{array}{l}\text { CHM } \\
\text { formulations }\end{array}$ & Object & Effect/Mechanism & Controls & $\begin{array}{l}\text { Minimal active } \\
\text { dosage }\end{array}$ & References \\
\hline $\mathrm{BHT}$ & $\begin{array}{l}\mathrm{MCAO} / \mathrm{R} \text {-induced rats (in } \\
\text { vivo); } \mathrm{H}_{2} \mathrm{O}_{2} / \mathrm{R} \text {-induced } \\
\text { HUVECs (in vitro) }\end{array}$ & $\begin{array}{l}\text { inhibits inflammation by regulating } \\
\text { decreasing proinflammatory cytokines (IL- } \\
6, \text { TNF- } \alpha \text { ) and increasing anti- } \\
\text { inflammatory cytokine (IL-10); maintains } \\
\text { the BBB integrity by inhibiting the } \\
\text { activation of the HIF-1 a/NEGF pathway } \\
\text { and stabilizing ion channel of } \beta \text {-ENaC in } \\
\text { brain; promotes angiogenesis by } \\
\text { modulating SIRT1NEGF and Nox4/ROS } \\
\text { signaling pathways }\end{array}$ & $\begin{array}{l}\text { sham-operated } \\
\text { (negative control) }\end{array}$ & $\begin{array}{l}0.5 \mathrm{~g} / \mathrm{kg} \text { (i.g.); } \\
10 \mathrm{mg} / \mathrm{ml} \\
\text { (incubate) }\end{array}$ & $\begin{array}{l}\text { Chen K. Y. et al. (2020); Chen } \\
\text { Z. Z et al. (2019); Zheng et al. } \\
\text { (2018); Shen et al. (2020) }\end{array}$ \\
\hline HJT & $\begin{array}{l}\text { MCAO/R-induced rats (in } \\
\text { vivo); OGD/R-induced } \\
\text { cerebral cortical neuron } \\
\text { (in vitro) }\end{array}$ & $\begin{array}{l}\text { inhibits apoptosis by activating the PI3K/ } \\
\text { AKT signaling pathway and HIF-1 } 1 \alpha \text {; } \\
\text { induces protective autophagy by } \\
\text { regulating MAPK-mTOR signaling } \\
\text { pathway }\end{array}$ & $\begin{array}{l}\text { sham-operated } \\
\text { (negative control) }\end{array}$ & $\begin{array}{l}2.5 \mathrm{~g} / \mathrm{kg} \text { (i.g.); } \\
0.1 \mathrm{mg} / \mathrm{ml} \\
\text { (incubate) }\end{array}$ & $\begin{array}{l}\text { Zhang Q. et al. (2014); Wang } \\
\text { et al. (2013) }\end{array}$ \\
\hline TST & $\begin{array}{l}\text { MCAO/R-induced rats (in } \\
\text { vivo) }\end{array}$ & $\begin{array}{l}\text { inhibits inflammation, apoptosis by } \\
\text { regulating the expressions of HIF-1a, } \\
\text { TNF- } \alpha \text {, iNOS and Caspase- } 3 \text {; protects Cl/ } \\
\text { RI by modulating the expressions of brain- } \\
\text { derived neurotrophic factor and p53 }\end{array}$ & $\begin{array}{l}\text { sham-operated } \\
\text { (negative control) }\end{array}$ & $0.7 \mathrm{~g} / \mathrm{kg}$ (i.g.) & $\begin{array}{l}\text { Wu C. J. et al. (2011); Wu, } \\
\text { (2018); Fan et al. (2015) }\end{array}$ \\
\hline NTF & $\begin{array}{l}\text { MCAO/R-induced rats (in } \\
\text { vivo) }\end{array}$ & $\begin{array}{l}\text { inhibits ferroptosis through adjusting the } \\
\text { TFR1/DMT1 and SCL7A11/GPX4 } \\
\text { signaling pathways and the expression of } \\
\text { Ferroportin to balance iron levels }\end{array}$ & $\begin{array}{l}\text { sham-operated } \\
\text { (negative control); } \\
\text { deferiprone (positive } \\
\text { control) }\end{array}$ & 0.7 g/kg (i.g.) & $\begin{array}{l}\text { Liao et al. (2015); Lan et al. } \\
\text { (2020) }\end{array}$ \\
\hline QY & $\begin{array}{l}\text { MCAO/R-induced mice (in } \\
\text { vivo) }\end{array}$ & $\begin{array}{l}\text { inhibits inflammation by modulating IL- } 1 \beta \text {, } \\
\text { IL-6, TNF- } \alpha \text {, NF- } k B \text { p } 65 \text {, TGF- } \beta 1 \text {, TLR4, } \\
\text { and Interferon- } \gamma\end{array}$ & $\begin{array}{l}\text { sham-operated } \\
\text { (negative control); } \\
\text { edaravone (positive } \\
\text { control) }\end{array}$ & 5.69 mg/ml (i.g.) & Wang Y. et al., 2020 \\
\hline SKD & $\begin{array}{l}\text { MCAO/R-induced rats (in } \\
\text { vivo) }\end{array}$ & $\begin{array}{l}\text { mitigates apoptosis and oxidation by } \\
\text { upregulating SOD and GSHPx levels, } \\
\text { downregulating iNOS, TNOS, and } \\
\text { Caspase-3 expressions }\end{array}$ & $\begin{array}{l}\text { sham-operated } \\
\text { (negative control); } \\
\text { nimodipine (positive } \\
\text { control) }\end{array}$ & $0.7 \mathrm{~g} / \mathrm{kg}$ (i.g.) & Chen et al. (2014) \\
\hline GGD & $\begin{array}{l}\text { MCAO/R-induced rats (in } \\
\text { vivo) }\end{array}$ & $\begin{array}{l}\text { mitigates oxidation by regulating SOD, } \\
\text { MDA and GSHPx levels; inhibits } \\
\text { inflammation by modulating NF- } \mathrm{kB} \\
\text { signaling pathway; inhibits ferroptosis by } \\
\text { regulating Poly (ADP-ribose) (PAR) } \\
\text { polymerase-1 (PARP-1)/apoptosis- } \\
\text { inducing factor (AIF) signaling pathway; } \\
\text { mitigates intracellular } \mathrm{Ca}^{2+} \text { overload by } \\
\text { regulating the concentration of } \mathrm{Ca}^{2+}\end{array}$ & $\begin{array}{l}\text { sham-operated } \\
\text { (negative control) }\end{array}$ & $3.6 \mathrm{~g} / \mathrm{kg}$ (i.g.) & $\begin{array}{l}\text { Zhang S. et al. (2015); Hu et al. } \\
\text { (2015); Nan et al. (2020); Hu } \\
\text { et al. (2018) }\end{array}$ \\
\hline MYF & $\begin{array}{l}\text { MCAO/R-induced rats (in } \\
\text { vivo) }\end{array}$ & $\begin{array}{l}\text { inhibits ferroptosis by regulating the } \\
\text { expressions of Bcl-2/Bax, Cyt-c, } \\
\text { Caspase-9/-3/-7; mitigates oxidation by } \\
\text { modulating the content of SOD, LDH, } \\
\text { CAT, MDA, and GSH-PX; inhibits } \\
\text { autophagy by activating AMPK/mTOR } \\
\text { signaling pathway }\end{array}$ & $\begin{array}{l}\text { sham-operated } \\
\text { (negative control); } \\
\text { nimodipine (positive } \\
\text { control) }\end{array}$ & 58 mg/kg (i.g.) & $\begin{array}{l}\text { Zhao et al. (2016); Wang et al. } \\
\text { (2017); Chen et al. (2016) }\end{array}$ \\
\hline STL & $\begin{array}{l}\text { MCAO/R-induced rats (in } \\
\text { vivo) }\end{array}$ & $\begin{array}{l}\text { exerts anti-inflammation and } \\
\text { antiapoptosis effects through activating } \\
\text { the SIRT1 signaling pathway }\end{array}$ & $\begin{array}{l}\text { sham-operated } \\
\text { (negative control) }\end{array}$ & 5.7 ml/kg (i.g.) & Mei et al. (2017) \\
\hline
\end{tabular}

\subsubsection{Gastrodia elata Blume [Orchidaceae; Gastrodiae Rhizome]}

Gastrodia elata Blume [Orchidaceae; Gastrodiae rhizome] is a famous CHM that has been traditionally used for the treatment and prevention of CI/RI for centuries (Liu et al., 2018). Gastrodin (GAS), a phenolic extracted from Gastrodia elata Blume [Orchidaceae; Gastrodiae rhizome], was revealed to improve antioxidant, anti-inflammatory, and antiapoptotic activities in $\mathrm{CI} / \mathrm{RI}$ models. In $\mathrm{MCAO} / \mathrm{R}$-induced rats and $\mathrm{H}_{2} \mathrm{O}_{2} / \mathrm{R}$-induced cells, some researchers indicate that GAS suppressed expressions of Caspase- 3 and Bax; upregulated the amount of SOD and the expressions of HO-1 and SOD1; and decreased levels of Bcl-2, TNF- $\alpha$, IL- $1 \beta$, and MDA. Moreover, it significantly activated the Akt/Nrf2 signaling pathway (Peng et al., 2015; Liu et al., 2016; Shi et al., 2018). It is worth noting that the adverse drug reaction (ADR) or event (ADE) of GAS cannot be ignored because there is a retrospective study with ADR or ADE induced by GAS (Zheng et al., 2018). Therefore, it is necessary to work out a more detailed 
TABLE 3 | Recent advances in protective mechanisms of $\mathrm{CHM}$ preparations for $\mathrm{Cl} / \mathrm{Rl}$.

\begin{tabular}{|c|c|c|c|c|c|}
\hline $\begin{array}{l}\text { CHM } \\
\text { preparations }\end{array}$ & Object & Effect/Mechanism & Controls & $\begin{array}{l}\text { Minimal active } \\
\text { dosage }\end{array}$ & References \\
\hline ANW & MCAO/R-induced rats (in vivo) & $\begin{array}{l}\text { protects BBB integrity by inhibiting } \\
\text { the activations of MMP- } 2 \text { and MMP- } \\
9 \text { and upregulating the expressions } \\
\text { of } Z \mathrm{O}-1 \text { and claudin- } 5\end{array}$ & $\begin{array}{l}\text { sham-operated group } \\
\text { (negative control) }\end{array}$ & 257 mg/kg (i.g.) & $\begin{array}{l}\text { Tsoi et al. (2019a); Tsoi et al. } \\
\text { (2019b) }\end{array}$ \\
\hline QLI & MCAO/R-induced rats (in vivo) & $\begin{array}{l}\text { inhibits apoptosis and inflammation } \\
\text { by modulating the AMPK/NLRP3 } \\
\text { signaling pathway; protects BBB } \\
\text { integrity by regulating expression of } \\
\text { TJs and HIF-1a/MMP-9 }\end{array}$ & $\begin{array}{l}\text { sham-operated group } \\
\text { (negative control) }\end{array}$ & 3 ml/kg (i.p.) & $\begin{array}{l}\text { Ma et al. (2019); Zhang S. } \\
\text { et al. (2020) }\end{array}$ \\
\hline TLC & $\begin{array}{l}\text { MCAO/R-induced rats (in } \\
\text { vivo); Human cardiac } \\
\text { microvascular endothelial cells } \\
\text { (HCMECs) (in vitro) }\end{array}$ & $\begin{array}{l}\text { inhibits apoptosis by regulating the } \\
\text { PI3K/Akt signaling pathway and } \\
\text { Connexin 43/Calpain II/Bax/ } \\
\text { Caspase-3 signaling pathway; } \\
\text { enhances neurogenesis and } \\
\text { angiogenesis; inhibits inflammation } \\
\text { by downregulating AQP4, TLR4, } \\
\text { NF-kB, and TNF- } \alpha \text { expressions }\end{array}$ & $\begin{array}{l}\text { sham-operated group } \\
\text { (negative control); nimodipine } \\
\text { (positive control) }\end{array}$ & $\begin{array}{l}0.5 \mathrm{~g} / \mathrm{kg} \text { (i.g.); } \\
200 \mu \mathrm{g} / \mathrm{ml} \\
\text { (incubate) }\end{array}$ & $\begin{array}{l}\text { Yu et al. (2019); Cheng et al. } \\
\text { (2017); Li et al. (2015); Cai } \\
\text { et al. (2016) }\end{array}$ \\
\hline $\mathrm{DHI}$ & $\begin{array}{l}\text { MCAO/R-induced rats (in } \\
\text { vivo); } \mathrm{H} 2 \mathrm{O} 2 / \mathrm{R} \text {-induced } \\
\text { mouse Neuro-2A cells (in vitro) }\end{array}$ & $\begin{array}{l}\text { inhibits apoptosis by regulating } \\
\text { PI3K/Akt signaling pathway; } \\
\text { prevents brain damage by activating } \\
\text { Nrf2/ARE signaling pathway; inhibits } \\
\text { inflammation by regulating NF-kB } \\
\text { signaling pathway; improves the } \\
\text { relative mitochondrial reductase } \\
\text { activity of the cultured neurons }\end{array}$ & $\begin{array}{l}\text { sham-operated group } \\
\text { (negative control); edaravone/ } \\
\text { ginaton group (positive } \\
\text { control) }\end{array}$ & 0.72 ml/kg (i.p.) & $\begin{array}{l}\text { Feng et al. (2020); Guo, H. } \\
\text { et al. (2014); Jiang and Lian } \\
\text { et al. (2015); Orgah J. Q. } \\
\text { et al. (2019) }\end{array}$ \\
\hline PTH & MCAO/R-induced rats (in vivo) & $\begin{array}{l}\text { inhibits apoptosis and inflammation } \\
\text { by regualting Cyt-c, Bax, P53, } \\
\text { Caspase-3/-9 Bcl-xl, AKT, and } \\
\text { GSK-3 } \beta\end{array}$ & $\begin{array}{l}\text { sham-operated group } \\
\text { (negative control) }\end{array}$ & 257 mg/kg (i.g.) & Zhang X. et al., 2018 \\
\hline ZFG & $\begin{array}{l}\text { MCAO/R-induced rats (in } \\
\text { vivo); OGD/R-induced } \\
\text { BMECs (in vitro) }\end{array}$ & $\begin{array}{l}\text { protects angiogenesis via Notch } \\
\text { and Wnt signaling pathways }\end{array}$ & $\begin{array}{l}\text { sham-operated group } \\
\text { (negative control) }\end{array}$ & 10 ml/kg (i.g.) & Huang et al. (2019) \\
\hline $\mathrm{HHS}$ & $\begin{array}{l}\text { MCAO/R-induced rats (in } \\
\text { vivo); OGD/R-induced } \\
\text { HUVECs (in vitro) }\end{array}$ & $\begin{array}{l}\text { promotes angiogenesis by } \\
\text { regulating HIF-1 } \alpha \text { NEGF and } \\
\text { stromal cell derived factor-1 (SDF- } \\
\text { 1)/cxc chemokine receptor } 4 \\
\text { (CXCR4) signaling pathways; } \\
\text { improves neurological function and } \\
\text { survival by activating BDNF/PI3K } \\
\text { Akt signaling pathways }\end{array}$ & $\begin{array}{l}\text { sham-operated group } \\
\text { (negative control) and ginaton } \\
\text { group (positive control) }\end{array}$ & $5.1 \mathrm{~g} / \mathrm{kg}$ (i.g.) & $\begin{array}{l}\text { Xiang et al. (2019); Chang } \\
\text { et al. (2016); Zhang Q. et al. } \\
\text { (2012) }\end{array}$ \\
\hline YFPI & $\begin{array}{l}\text { MCAO/R-induced mice (in } \\
\text { vivo); OGD/R-induced bEnd.3 } \\
\text { cells (in vitro) }\end{array}$ & $\begin{array}{l}\text { improves BBB dysfunction by } \\
\text { increasing expressions of tight } \\
\text { junction proteins and inhibiting the } \\
\text { NF-kB signaling pathway }\end{array}$ & $\begin{array}{l}\text { sham-operated group } \\
\text { (negative control) and } \\
\text { XueShuanTong Injection } \\
\text { group (positive control) }\end{array}$ & $\begin{array}{l}336 \mathrm{mg} / \mathrm{kg} \text { (i.p.); } \\
100 \mu \mathrm{g} / \mathrm{ml} \\
\text { (incubate) }\end{array}$ & $\begin{array}{l}\text { Cao et al. (2016); Pan et al. } \\
\text { (2020) }\end{array}$ \\
\hline SMl & MCAO/R-induced rats (in vivo) & $\begin{array}{l}\text { attenuates autophagy by } \\
\text { modulating the AMPK, mTOR, and } \\
\text { JNK pathways; maintains BBB } \\
\text { integrity by regulating the TJ- } \\
\text { associated proteins }\end{array}$ & $\begin{array}{l}\text { sham-operated group } \\
\text { (negative control); 3-meth- } \\
\text { yladenine group (positive } \\
\text { control) }\end{array}$ & 1.42 g/kg (i.p.) & Yang et al. (2016); Xu (2019) \\
\hline XSTI & $\begin{array}{l}\text { MCAO/R-induced rats (in } \\
\text { vivo); OGD/R-induced SH- } \\
\text { SY5Y cells (in vitro) }\end{array}$ & $\begin{array}{l}\text { exerts long-term neuroprotection by } \\
\text { inhibiting the ROCKII pathway }\end{array}$ & $\begin{array}{l}\text { sham-operated group } \\
\text { (negative control) }\end{array}$ & 20 mg/kg (i.v.) & Zhou et al. (2021) \\
\hline
\end{tabular}

toxicity study of GAS according to the International Council for Harmonization safety guidelines.

\subsubsection{Carthamus tinctorius L (CTL) [Asteraceae; Carthami Flos]}

Carthamus tinctorius L (CTL) [Asteraceae; Carthami Flos] has functions on removing blood stasis and promoting blood circulation and has been used for treating coronary heart disease and cerebral thrombosis. It ameliorates $\mathrm{CI} / \mathrm{RI}$ rats with antiapoptosis, antioxidant, and anti-inflammatory effects. CTL regulates the expressions of Bcl-2, Bax, Caspase-3, and MMP-9 (Chang et al., 2020); changes the content of TNF- $\alpha$ and IL-1 $\beta$ (Fu et al., 2016); and modulates the TNF- $\alpha$ /MAPK (Wang et al., 2012) and JAK2/STAT3 signaling pathways (Hu et al., 2017). Hydroxysafflor yellow A (HSYA), the active chalcone glycoside extracted from CTL, can alleviate CI/RI by inhibiting pyroptosis in a 
multitarget way. HSYA downregulates the levels of NLRP3 and Caspase-1, the dissociation of GSDMD, and the generation of the pore. What is more, HSYA restrains activation of the NF- $\kappa B$ pathway to reduce the production of pro-inflammatory factors, such as IL-1 $\beta$ and IL-18 (Tan et al., 2020). A study reported by Chen and colleagues reveals that HSYA protects against CI/RI by an anti-apoptotic effect through modulating the PI3K/Akt signaling pathway in rats (Chen et al., 2013).

\subsubsection{Astragalus mongholicus Bunge [Fabaceae; Astragali Radix]}

Astragalus mongholicus Bunge [Fabaceae; Astragali radix] is recorded in "Shennong's Classic of Materia Medica" with the effects of stagnation and diuresis, which are commonly used in clinical trials of CHM. Astragaloside IV (AIV) and astragaloside VI (AVI) are natural saponins that are abundant in Astragalus mongholicus Bunge [Fabaceae; Astragali radix], which have neuroprotective effects against $\mathrm{CI} / \mathrm{RI}$ through reducing oxidative stress, apoptosis, and inflammation (Jia et al., 2014; Wang et al., 2017). In a study by Li et al. (2019), to investigate the parthanatos in CI/RI, the author shows that AIV activates AKT to promote HK-II binding to mitochondria and changes mitochondria's structure. The structural and functional integrity of mitochondria is essential for AIV to protect neuronal survival from brain damage. There is a study reporting that AIV also alleviates CI/RI by activating the Janus kinase 2 and signal transducer and activator of transcription 3 (JAK2/STAT3) signaling pathway (Xu et al., 2020). AVI activated the EGFR/MAPK signaling pathway to promote the proliferation and neurogenesis of neural stem cells and further to improve the repair of neurological function in the $\mathrm{MCAO} / \mathrm{R}$ model of rats (Chen X et al., 2019).

\subsubsection{Pueraria montana var. Lobata (Willd.) Maesen and S.M.Almeida Ex Sanjappa \& Predeep [Fabaceae; Puerariae lobatae Radix]}

Pueraria montana var. lobata (Willd.) Maesen and S.M.Almeida ex Sanjappa \& Predeep [Fabaceae; Puerariae lobatae radix] is a common component of health products and medicines. Puerarin is a major iso-flavonoid extracted from this leguminous plant. In vivo study investigating the effect of puerarin on MCAO/ $\mathrm{R}$-induced rats shows that puerarin improves neurological deficit and reduces infarct size and brain water content in CI/ RI rats. The probable mechanism proposed ias the involvement of puerarin in the TLR4/NF- $\mathrm{BB}$ signaling pathway (Zhou F. et al., 2014). In vitro study reports that puerarin and catalpol protect the brain from ischemia via protecting endothelial cells from apoptosis. This protection is related to HIF-1a that is dependent on the ERK and PI3K/AKT/mTOR signaling pathways (Liu Y. et al., 2017).

\section{CHM FORMULATIONS FOR CI}

\subsection{Buyang Huanwu Tang}

Buyang Huanwu Tang (BHT) is a classic formula that has been used in patients with ischemic stroke for many years. It originates from the old record "Yi Lin Gai Cuo" and was compiled by Qingren Wang, a famous doctor in the Qing dynasty. BHT contains six botanical $\operatorname{drug}(\mathrm{s})$, including Astragalus mongholicus Bunge [Fabaceae; Astragali radix], Angelica sinensis (Oliv.) Diels [Apiaceae; Angelicae sinensis radix], Paeonia lactiflora Pall [Paeoniaceae; Paeoniae radix rubra], Conioselinum anthriscoides 'Chuanxiong' [Apiaceae; Chuanxiong rhizoma], Prunus persica (L.) Batsch [Rosaceae; Persicae semen] and Carthamus tinctorius L [Asteraceae; Carthami Flos]. Reportedly, BHT can facilitate neurorehabilitation following a CI insult through improving synaptic plasticity (Pan et al., 2017). Chen et al. randomly divided $108 \mathrm{SD}$ rats into sham operation, MCAO/R, MCAO/ $\mathrm{R}+\mathrm{BHT}$, and MCAO/R + edaravone groups. They found that BHT treatment decreased cerebral edema and rat neurological function scores and reduced brain infarct volume by reducing the protein and mRNA levels of HIF- $1 \alpha$ and VEGF (Chen Z. Z et al., 2019). In the study by Chen K. Y. et al. (2020), the authors reveal that BHT powdered product exhibited therapeutic efficacy for experimental stroke via reducing the expressions of TNF- $\alpha$ and IL- 6 and increasing the expressions of TGF- $\beta$ and IL-10. To explore the BHT effects on angiogenesis and neuroprotection after $\mathrm{CI} / \mathrm{RI}$, some researchers made $\mathrm{H}_{2} \mathrm{O}_{2}$ /R-induced oxidative stress in human umbilical vein endothelial cells (HUVECs) and $\mathrm{MCAO} / \mathrm{R}$-induced rats. The data show that $\mathrm{BHT}$ promotes angiogenesis after CI/RI by controlling the Nox4/ROS and SIRT1/VEGF signaling pathways (Zheng et al., 2018; Shen et al., 2020).

\subsection{Hulian Jiedu Tang}

Hulian Jiedu Tang (HJT), an ancient antipyretic and detoxifying formula, is first recorded in the book "Wai Tai Mi Yao" by Wang Tao during the Tang Dynasty. It consists of Coptis chinensis Franch [Ranunculaceae; Coptidis rhizoma], Scutellaria baicalensis Georgi [Lamiaceae; Scutellariae radix], Phellodendron amurense Rupr [Rutaceae; Phellodendri chinensis cortex], and Gardenia jasminoides J. Ellis [Rubiaceae; Gardeniae fructus] in a ratio of 3: 2:2:3. HJT is reported to improve antioxidant, anti-inflammatory, and antiapoptosis and regulate energy metabolism and mitochondrial function activities in CI/RI models. Zhu et al. applied metabolomics analysis and basic pathophysiology to evaluate the mechanisms of HJT against CI comprehensively. The data show that HJT can modulate metabolic stress, regulate the glutamate/GABA-glutamine cycle, and maintain cholinergic neuron function to modulate cerebral blood flow, regulate neuroinflammation and enhance memory (Zhu et al., 2018). Furthermore, HJT relieved $\mathrm{CI} / \mathrm{RI}$ and neuronal apoptosis in MCAO/R-induced SD rats and OGD/R-induced cerebral cortical neurons through activating the PI3K/AKT signaling pathway and HIF-1a (Zhang Q. et al., 2014). In CI/RI rats, HJT induced protective autophagy by elevating the levels of Beclin-1 and LC3-II/LC3-I, inhibiting the mTOR via MAPK signaling pathway; in the other words, via restraining the phosphorylation of JNK and P38 and promoting the activation of ERK (Wang et al., 2013). A reporter explored the effects of the total alkaloids, flavonoids, and iridoids from HJT against CI/RI, and the result suggests that the total iridoids of HJT promote 
angiogenesis by modulating VEGF, Ang-1/-2, and TGF- $\beta 1$; alkaloids were partially correlated with increased phosphorylation of AKT and GSK-3 $\beta$; and flavonoids might be associated with the regulation of AKT, GSK-3 $\beta$, mRNA, and Ang-1 protein levels (Zou et al., 2016). Berberine, baicalin, and geniposide (BBG), the three major ingredients in $\mathrm{HJT}$, exhibit outstanding antioxidative effects for MCAO/ R-injury rats by modulating the activities of SOD, CAT, and GPX (Fu et al., 2019).

\subsection{Taohong Siwu Tang}

Taohong Siwu Tang (TST), consists of Prunus persica (L.) Batsch [Rosaceae; Persicae semen], Carthamus tinctorius L [Asteraceae; Carthami Flos], Angelica sinensis (Oliv.) Diels [Apiaceae; Angelicae sinensis radix], Conioselinum anthriscoides 'Chuanxiong' [Apiaceae; Chuanxiong rhizoma], Rehmannia glutinosa (Gaertn.) DC [Orobanchaceae; Rehmanniae radix], and Paeonia lactiflora Pall [Paeoniaceae; Paeoniae radix rubra] and has been used clinically for the treatment of cerebrovascular diseases due to its effect of promoting blood circulation. TST reduces infarct volume in CI/RI rats through inhibiting inflammatory responses, apoptosis formation, and platelet activation via regulating the expressions of HIF-1 $\alpha$, TNF- $\alpha$, iNOS, and Caspase-3 (Wu C. J. et al., 2011). TST modulates the expressions of brain-derived neurotrophic factor and p53 to play a role in treating and protecting CI/RI (Fan et al., 2015; Wu, 2018).

\subsection{Nao Tai Fang}

Nao Tai Fang (NTF) is a CHM formulation that improves blood circulation. The substance is formulated by modifying BHT and consists of Astragalus mongholicus Bunge [Fabaceae; Astragali Radix], Conioselinum anthriscoides 'Chuanxiong' [Apiaceae; Chuanxiong rhizoma], Pheretima aspergillum (E. Perrier) [Megascolecidae; Pheretima], and Bombyx mori Linnaeus [Bombycidae; Bombyx batryticatus]. Some studies demonstrate that NTF is clinically effective for the treatment of CI/RI, and the therapeutic mechanism is correlated with neuron ferroptosis. In an experimental investigation of NTF extraction on rat models of $\mathrm{CI} / \mathrm{RI}$, the author reports an increased expression of iron in the hippocampal CA2 region in the treatment group (Liao et al., 2015), suggesting the protective effect of NTF on the neurons by increasing the expression of iron and promoting neuronal iron efflux in CI/RI models. Furthermore, the recent study indicates that NTF extraction exerts a neuroprotective effect by promoting the expression of SLC7A11, intracellular GSH synthesis, and GPX4 activity and downregulating TFR1 and DMT1 protein levels in the MCAO/R-induced rats (Lan et al., 2020).

\subsection{Qishen Yiqi}

Qishen Yiqi (QY), composed of Astragalus mongholicus Bunge [Fabaceae; Astragali Radix], Salvia miltiorrhiza Bunge [Lamiaceae; Salviae miltiorrhizae radix et rhizoma], Panax notoginseng (Burkill) F.H.Chen [Araliaceae; Notoginseng radix et rhizoma], and Dalbergia odorifera T.C.Chen [Fabaceae; Dalbergiae odoriferae lignum], exerts the function of Qi tonifying and blood activating. The authors use the combination method of the pharmacology network and experimental verification to excavate the potential of QY about the reduction of neuroinflammatory response in a stroke subject. The result shows that the core targets associated with neuroinflammatory response were as follows: IL-1 $\beta$, IL-6, TNF- $\alpha$, NF- $\kappa$ B p65, TGF- $\beta 1$, TLR4, and Interferon- $\gamma$ (IFNG$\gamma)$. IFNG- $\gamma$ is an inflammatory mediator released from $\mathrm{CD}^{+}$and $\mathrm{CD}^{+}$T-lymphocytes as well as natural killer (NK) cells (Wang Y. et al., 2020).

\subsection{Shengnao Kang Decoction}

Shengnao Kang Decoction (SKD) includes 15 kinds of CHM as follows: Conioselinum anthriscoides "Chuanxiong" [Apiaceae; Chuanxiong rhizoma], Salvia miltiorrhiza Bunge [Lamiaceae; Salviae miltiorrhizae radix et rhizoma], Gastrodia elata Blume [Orchidaceae; Gastrodiae rhizome], Panax notoginseng (Burkill) F.H.Chen [Araliaceae; Notoginseng radix et rhizoma], Astragalus mongholicus Bunge [Fabaceae; Astragali Radix], Pueraria montana var. lobata (Willd.) Maesen and S.M.Almeida ex Sanjappa and Predeep [Fabaceae; Puerariae lobatae radix], Paeonia lactiflora Pall [Paeoniaceae; Paeoniae radix rubra], Leonurus japonicus houtt. [Lamiaceae; Leonuri herba], Uncaria rhynchophylla (Miq.) Miq. [Rubiaceae; Uncariae ramulus cum uncis], Sophora flavescens Aiton [Fabaceae; Sophora flavescentis radix], Whitmania pigra Whitman [Hirudinidae; Hirudo], Eupolyphaga sinensis Walker [eupolyphaga; Eupolyphaga steleophaga], Bombyx mori Linnaeus [Bombycidae; Bombyx batryticatus], Pheretima aspergillum (E. Perrier) [Megascolecidae; Pheretima], and Scolopendra subspinipes mutilans L. Koch [Scolopendridae; Scolopendra]. It has efficacies of activating blood circulation and dissipating blood stasis, dredging meridians and collaterals. Dang et al. carried out a study on the antithrombotic effect of reduced SKD (RSKD), which is composed of Conioselinum anthriscoides "Chuanxiong" [Apiaceae; Chuanxiong rhizoma], Salvia miltiorrhiza Bunge [Lamiaceae; Salviae miltiorrhizae radix et rhizoma], Astragalus mongholicus Bunge [Fabaceae; Astragali Radix], Pueraria montana var. lobata (Willd.) Maesen and S.M.Almeida ex Sanjappa and Predeep [Fabaceae; Puerariae lobatae Radix], Paeonia lactiflora Pall [Paeoniaceae; Paeoniae radix rubra], and Panax notoginseng (Burkill) F.H.Chen [Araliaceae; Notoginseng radix et rhizoma]. RSKD had activities of anticoagulation, the regulation of active substances in vascular endothelium, and maintaining the balance of thromboxane A2 (TXA2) and prostaglandins I2 (PGI2) in CI/ $\mathrm{RI}$ rats (Dang et al., 2015). In a study, the treatment with SKD on $\mathrm{MCAO} / \mathrm{R}$ rats could diminish the levels of MDA, iNOS, and TNOS and increase the SOD and GPX activities (Chen et al., 2014).

\subsection{Gualou Guizhi Decoction}

Gualou Guizhi decoction (GGD), written by Zhang Zhongjing in "JinKui YaoLue" during the Eastern Han Dynasty, consists of six kinds of CHM: Trichosanthes kirilowii Maxim. [Cucurbitaceae; Trichosanthis fructus], Neolitsea cassia (L.) Kosterm. [Lauraceae; Cinnamomi ramulus], Paeonia lactiflora Pall [Paeoniaceae; Paeoniae radix rubra], Zingiber officinale Roscoe 
[Zingiberaceae; Zingiberis rhizoma], Ziziphus jujuba Mill [Rhamnaceae; Jujubae fructus], and Glycyrrhiza uralensis Fisch. ex DC [Fabaceae; Glycyrrhizae radix et rhizoma]. It has long been used to treat $\mathrm{CI}$ in a clinical setting in China. Some studies suggest that GGD has a neuroprotective effect on CI/RI models, and this effect is likely to be associated with its function as an antioxidant (by reducing MDA level and increasing GSH and SOD activities) (Zhang S. et al., 2015), anti-inflammatory (by regulating the NF- $\kappa B$ signaling pathway) (Hu et al., 2015), and antiapoptosis (by regulating protein expression and translocation in the PARP-1/AIF signaling pathway) (Nan et al., 2020; Hu et al., 2018). After the oral administration of $10 \mathrm{ml} / \mathrm{kg} \mathrm{GGD}$ to MCAO/ $\mathrm{R}$ model rats for 7 days, $\mathrm{Hu}$ et al. find that GGD can be against intracellular $\mathrm{Ca}^{2+}$ overload. Due to the effectiveness, GGD was made into the Gualou Guizhi granule. The Gualou Guizhi granule, as a hospital preparation of the second people's Hospital of Fujian province (Min drug system approval No. S20130001) (Yang et al., 2012), is widely used for treating muscular spasticity following CI/RI. Zhang et al. find that Gualou Guizhi Granule protects oxidative injury by activating the Nrf2/antioxidant response element (ARE) signaling pathway in MCAO/R-induced rats and $\mathrm{H}_{2} \mathrm{O}_{2}$ /R-induced PC12 cells (Zhang Y. et al., 2018).

\subsection{Muxiang You Fang}

Muxiang You Fang (MYF), one of the classical Traditional Hui Medicine (THM) formulas, was recorded in a Chinese Hui medical classic "Hui Hui Yao Fang." It is regared to be beneficial in the treatment of CI/RI with consists of Aucklandia costus Falc. [Asteraceae; Aucklandiae radix], Piper nigrum L [Piperaceae; Piperis fructus], Euphorbia pekinensis Rupr. [Euphorbiaceae; Euphorbiae pekinensis radix], Phoca vitulina (L.) [Otariidae; Phocae vitulina testes et penis], and Asarum sieboldii Miq [Aristolochiaceae; Asari radix et rhizoma]. The group of Zhao QP found that MYF has potential neuroprotective activities by modulating the ratio between Bcl-2 and Bax (Zhao et al., 2016); promoting the protein expressions of Cyt-c and Caspase-3/-9 (Zhao et al., 2016); increasing the content of SOD, LDH, CAT, MDA, and GSH-PX (Wang et al., 2017); and activating the AMPK/mTOR signaling pathway (Chen, 2016) in treatment of CI/RI rats. THM, a branch of CHM, is efficient in the treatment of encephalopathy, especially stroke.

\subsection{Shuan Tong Ling}

Shuan Tong Ling (STL), a formula used to treat brain diseases, is composed of Pueraria montana var. lobata (Willd.) Maesen and S.M.Almeida ex Sanjappa and Predeep [Fabaceae; Puerariae lobatae radix], Salvia miltiorrhiza Bunge [Lamiaceae; Salviae miltiorrhizae radix et rhizoma], Curcuma longa L. [Zingiberaceae; Curcumae longae rhizoma], Crataegus monogyna Jacq [Rosaceae; Crataegi fructus], Salvia chinensis Benth. [Lamiaceae; Salviae herba], Sinapis alba L [Brassicaceae; Sinapis semen], Astragalus mongholicus Bunge [Fabaceae; Astragali radix], Panax japonicus (T.Nees) C.A.Mey [Araliaceae; Panacis japonici rhizoma], Atractylodes macrocephala Koidz. [Asteraceae; Atractylodis macrocephalae rhizoma], Paeonia lactiflora Pall. [Paeoniaceae; Paeoniae radix rubra], Petroselinum crispum (Mill.) Fuss [Apiaceae; Bupleuri radix], Matricaria chamomilla L. [Asteraceae; Matricariae fols], Cyperus rotundus L. [Cyperaceae; Cyperi rhizoma], and Gastrodia elata Blume [Orchidaceae; Gastrodiae rhizome]. MCAO/ R-induced SD rats revealed that STL exerts anti-inflammatory and antiapoptosis effects through activation of the SIRT1 signaling pathway (Mei et al., 2017).

\section{CHM PREPARATIONS FOR CI/RI}

\subsection{Angong Niuhuang Wan}

Angong Niuhuang Wan (ANW), one of the representative prescriptions for treating $\mathrm{CI}$, originated from the "Wenbing Tiaobian" written by the physician Jutong $\mathrm{Wu}$ in the Qing Dynasty. It consists of Coptis chinensis Franch. [Ranunculaceae; Coptidis rhizoma], Scutellaria baicalensis Georgi [Lamiaceae; Scutellariae radix], Gardenia jasminoides J. Ellis [Rubiaceae; Gardeniae fructus], Curcuma aromatica Salisb. [Zingiberaceae; Curcumae radix], Bos taurus domesticus Gmeli [Bovidae; Bovis calculus], Bubalus bubalis Linnaeu [Bovidae; Bubali cornu], Moschus berezovskii Flerov [Cervidae; Moschus], Pteria martensii (Dunker) [Pteriidae; Margarita], cinnabaris (mineral drug), sulfur (mineral drug), and borneolum syntheticum (Guo Y. et al., 2014). The metaanalysis shows that ANW treatment improves the total response rate and neurologic deficit score among acute $\mathrm{CI}$ and intracerebral hemorrhage patients (Liu et al., 2019). A recent study uses MCAO/R-induced SD rats to explore the protective effects against $\mathrm{CI} / \mathrm{RI}$, and finds that the underlying mechanisms of ANW can be attributed to preserving BBB integrity by inhibiting the activation of MMP-2/-9 and upregulating the expressions of ZO-1 and claudin-5 (Tsoi et al., 2019a; Tsoi et al., 2019b). Even though there is a study reporting that sulfur and cinnabaris are essential components contributing to the neuroprotection of ANW with no hepatorenal toxicity in CI/ $\mathrm{RI}$, the arsenic- and mercury-containing ingredients in the formula still catch attention for causing health problems after consumption (Tsoi et al., 2019b). For safety and convenience, Zhang et al. extracted and purified components from ANW, removed cinnabaris and sulfur, and then made it to be an Angong Niuhuang (AN) sticker, which has the functions of activating blood circulation to dissipate blood stasis, cooling blood, and promoting the circulation of Qi to relieve pain. The more exciting thing is that the AN sticker combined with point application can improve cognitive function, promote the expression of $\mathrm{Bcl}-2$, and inhibit the expressions of Bax and p53 in the hippocampal CA1 area of MCAO/R-induced rats and present a protective effect on neuronal damage (Zhang D. et al., 2012; Zhang R. et al., 2015).

\subsection{Qingkai Ling Injection}

Qingkai Ling Injection (QLI) is a patented CHM that is approved by the China FDA to treat ischemic stroke (http://samr.cfda.gov. $\mathrm{cn} / \mathrm{WS} 01 / \mathrm{CL} 0412 /)$. It is derived from ANW, whose main ingredients include Panax ginseng C.A.Mey. [Araliaceae; Ginseng radix et rhizoma], Cornus mas L. [Cornaceae; Corni 
fructus], Scutellaria baicalensis Georgi [Lamiaceae; Scutellariae radix], Lonicera japonica Thunb [Caprifoliaceae; Lonicerae japonicae flos], and Gardenia jasminoides J. Ellis [Rubiaceae; Gardeniae fructus]. QLI is widely used for treating ischemic stroke with the effects of heat clearing and detoxifying, eliminating phlegm and freeing channels, tranquilizing and allaying agitation, recovering consciousness, and keeping the balance of Qi and blood (Wu et al., 2014). Ma et al. did an experiment on $\mathrm{MCAO} / \mathrm{R}$-induced SD rats to assess the protective effect of treatment. As a result, QLI significantly inhibited inflammation by decreasing the levels of pro-inflammatory cytokines (TNF- $\alpha$, IL- 6 , and IL-1 $\beta$ ) and increasing the levels of anti-inflammatory cytokines (IL-4 and IL-10) and inhibited apoptosis and oxidative stress by regulating the MAPK signaling pathway (Ma et al., 2019). Subsequently, the authors inspected the neuroprotective effect of refined QLI (made up of baicalin, geniposide, cholic acid, and hyodeoxycholic acid) against CI/RI and detected that the effect was related to its attenuation of brain damage and cell apoptosis and activation of glial cells by modulation of the PI3K/Akt, TNF, NOD-like receptor, NF- $\kappa \mathrm{B}$, and TLR signaling pathways (Ma C. et al., 2020). Zhang et al. combined a network pharmacology-based approach with the experiment to probe the biological mechanisms of QLI against CI on the BBB and found that QLI alleviated BBB dysfunction, regulated expression of TJ, and modulated HIF-1a/MMP-9 activation (Zhang S. et al., 2020). With good efficacy and pharmacological effect, QLI is considered the first choice for the clinical treatment of CI/RI. Nevertheless, there are ADR reports regarding QKL in clinical practice (Wang et al., 2010). The medical staff and researchers should pay special attention to this problem.

\subsection{Tongxin Luo Capsules}

Tongxin Luo Capsules (TLC) are extracted and concentrated from 12 kinds of CHM, such as Panax ginseng C.A.Mey. [Araliaceae; Ginseng radix et rhizoma], Paeonia lactiflora Pall. [Paeoniaceae; Paeoniae radix rubra], Santalum album L. [Santalaceae; Santali albi lignum], Dalbergia odorifera T.C.Chen [Leguminosae; Dalbergiae odoriferae lignum], Ziziphus jujuba Mill. [Rhamnaceae; Ziziphi jujubae semen], Whitmania pigra Whitman [Hirudinidae; Hirudo], Eupolyphaga sinensis Walker [eupolyphaga; Eupolyphaga steleophaga, Buthus martensii Karsch [Buthidae; Scorpio], Scolopendra subspinipes mutilans L. Koch [Scolopendridae; Scolopendra], Cryptotympana pustulata Fabriciu [Cicadidae; Cicadae periostracum], Boswellia carterii Birdw [Burseraceae; Olibanum], and Borneolum syntheticum. They were approved by the FDA of China in 1996 for the treatment of ischemic stroke. The current research suggests that TLC inhibits neuronal apoptosis by modulating the PI3K/Akt (Yu et al., 2019) and Connexin 43/Calpain II/Bax/Caspase-3 signaling pathways (Cheng et al., 2017); it inhibits postischemic inflammation by downregulating AQP4 expression and inhibiting the activation of high mobility group box 1 (one of the major inflammatory mediators), TLR4, NF- $\mathrm{B}$, and TNF- $\alpha$ (Cai et al., 2016). It also lowers cerebral infarct volume (Cheng et al., 2014), enhances neurogenesis as well as angiogenesis (Chen, 2016), and protects the brain from $\mathrm{BBB}$ disruption and increased TJ proteins ( $\mathrm{Li}$ et al., 2015).

\subsection{Dan Hong Injection}

Dan Hong injection (DHI), a well-established CHM prescription, consists of Salvia miltiorrhiza Bunge [Lamiaceae; Salviae miltiorrhizae radix et rhizoma] and Carthamus tinctorius L. [Asteraceae; Carthamus flos]. It is applied extensively in clinical practice and shows significant therapeutic effects to $\mathrm{CI} / \mathrm{RI}$ patients with the functions of promoting blood circulation and resolving stasis. Some researchers confirm the neuroprotective effects of DHI to CI/RI models and further discovered that these effects on the brain are partly generated by activating the PI3K-Akt (Feng et al., 2020), NF- $\kappa B$ (Jiang and Lian, 2015), and Nrf2/ARE signaling pathways (Guo H. et al., 2014) and enhancing the mitochondrial function (Orgah et al., 2019). As a CHM injection, DHI has a very good safety record, whose incidence rate of ADR was 3.50\% (Orgah et al., 2019).

\subsection{Pien Tze Huang}

Pien Tze Huang (PTH), a famous and precious Chinese patent medicine in China and Southeast Asian countries, was formulated by a royal physician in the Ming Dynasty. It is made up of Panax notoginseng (Burkill) F.H.Chen [Araliaceae; Notoginseng radix et rhizoma], Bos taurus domesticus Gmeli [Bovidae; Bovis calculus] (animal drug), Moschus berezovskii Flerov [Cervidae; Moschus] (animal drug), Panax ginseng C.A.Mey. [Araliaceae; Ginseng radix et rhizoma], and snake gall. PTH has activities of heat clearing and detoxifying, promoting blood circulation, reducing blood stasis and swelling, and relieving pain. It shows preventive effects on limiting the damage or injury caused by CI/RI in rats. PTH inhibits mitochondria-mediated neuronal apoptosis and attenuates inflammatory responses by downregulating cytosolic Cyt-c, Bax, P53, and Caspase-3/-9 levels and upregulating levels of mitochondrial Cyt-c, Bcl-xl, and phosphorylation of AKT and GSK-3 $\beta$ (Zhang $X$. et al., 2018).

\subsection{Zhong Feng Gao}

Zhong Feng Gao (ZFG), a hospital preparation with the effects of replenishing Qi, promoting blood circulation, dispersing blood stasis, and dredging collateral, consists of Curcuma longa L. [Zingiberaceae; Curcumae longae rhizoma], Conioselinum anthriscoides "Chuanxiong" [Apiaceae; Chuanxiong rhizoma], Astragalus mongholicus Bunge [Fabaceae; Astragali radix], Salvia miltiorrhiza Bunge [Lamiaceae; Salviae miltiorrhizae radix et rhizoma], Paeonia lactiflora Pall. [Paeoniaceae; Paeoniae radix rubra], and Glycyrrhiza uralensis Fisch. ex DC [Fabaceae; Glycyrrhizae radix et rhizoma]. Huang et al. (2019) prepared ZFG-medicated serum through administrating ZFG $(1 \mathrm{ml} / 100 \mathrm{~g})$ and adding DMEM containing $10 \% \mathrm{ZFG}$ medicated serum to the reoxygenation medium. The result demonstrates that treatment with ZFG-medicated serum markedly alleviated OGD/R-induced BMEC injury by protecting angiogenesis via the Notch and Wnt signaling pathways.

\subsection{Houshi Hei San}

Houshi Hei San (HHS), produced by Zhongjing Zhang for the treatment of $\mathrm{CI}$, is proved valuable in clinical practice, and it is 
composed of Chrysanthemum $\times$ morifolium (Ramat.) Hemsl. [Asteraceae; Chrysanthemi flos], Saposhnikovia divaricata (Turcz. ex Ledeb.) Schischk. [Apiaceae; Saposhnikoviae radix], Neolitsea cassia (L.). Kosterm [Lauraceae; Cinnamomi ramulus], Conioselinum anthriscoides "Chuanxiong" [Apiaceae; Chuanxiong rhizoma], Asarum sieboldii Miq.[ Aristolochiaceae; Asari radix et rhizoma], Platycodon grandiflorus (Jacq.) A. DC. [Campanulaceae; Platycodonis radix], Atractylodes macrocephala Koidz. [Asteraceae; Atractylodis macrocephalae rhizoma], Zingiber officinale Roscoe [Zingiberaceae; Zingiberis rhizoma], Angelica sinensis (Oliv.) Diels [Apiaceae; Angelicae sinensis radix], Panax ginseng C.A.Mey. (Araliaceae; Ginseng radix et rhizoma), Scutellaria baicalensis Georgi [Lamiaceae; Scutellariae radix], Poria cocos (Schw) Wolf [Polyporaceae; Poria], and Ostrea gigas Thunberg [Ostreidae; Ostreae concha]. HHS suppresses $\mathrm{CI} / \mathrm{RI}$ by promoting astrocyte activation and diminishing inflammatory factor expression (Zhang Q. et al., 2012). It promotes angiogenesis via upregulating the expression of HIF1a, VEGFA, and Ang-1 and downregulating the expression of Ang-2 (Xiang et al., 2019). HHS also can improve neurological function and survival by the activation of brain-derived neurotrophic factor (BDNF)/PI3K/Akt signaling pathways (Chang et al., 2016).

\subsection{Sheng Mai Injection}

Sheng Mai Injection (SMI), a derivative form of Sheng Mai San, consists of Panax ginseng C.A.Mey.(Araliaceae; Ginseng radix et rhizoma), Ophiopogon japonicus (Thunb.) Ker Gawl. [Asparagaceae; Ophiopogonis radix], and Schisandra chinensis (Turcz.) Baill. [Schisandraceae; Schisandrae chinensis fructus] and shows significant efficacy for the prevention and treatment of cerebrovascular diseases clinically. An in vivo study investigated the effect of SMI in MCAO/R rats at three oral doses: 1.42, 2.84, and $5.68 \mathrm{~g} / \mathrm{kg}$, which revealed that SMI attenuates CI/RI-induced autophagy by modulation of the AMPK, mTOR, and JNK signaling pathways (Yang et al., 2016). SMI maintained BBB integrity following CI/RI via regulating the expression and trafficking of TJ-associated proteins in lipid rafts (Xu, 2019).

\subsection{Yiqi Fumai Powder Injection}

Yiqi Fumai Powder Injection (YFPI), widely used for the treatment of cerebrovascular diseases, is made up of Panax ginseng C.A.Mey.(Araliaceae; Ginseng radix et rhizoma), Ophiopogon japonicus (Thunb.) Ker Gawl. [Asparagaceae; Ophiopogonis radix], and Schisandra chinensis (Turcz.) Baill. [Schisandraceae; Schisandrae chinensis fructus]. Some studies report that YFPI provides a neuroprotective effect against CI/ $\mathrm{RI}$ in mice by improving BBB dysfunction via increasing the expression of TJ proteins (Cao et al., 2016) and inhibiting the activation of the NF- $\kappa$ B signaling pathway (Pan et al., 2020).

\subsection{Xue Sai Tong Injection}

Xue Sai Tong Injection (XSTI) is also a classic CHM injection; its main constituent is saponins, which are extracted from Panax notoginseng (Burkill) F.H.Chen [Araliaceae; Notoginseng radix et rhizoma]. The research finds that XSTI reduces neurological dysfunction and pathological damage, promotes weight gain and synaptic regeneration, and reduces Nogo-A mRNA and protein levels by inhibiting the ROCKII pathway in models of MCAO-induced rats and OGD/R-induced SH-SY5Y cells (Zhou et al., 2021).

\section{DISCUSSION}

\subsection{Summary of Results}

In summary, CI/RI is a complex pathophysiological process. Different processes lead to different severity levels of CI/RI, among which the numerous factors and mechanisms interrelate with each other and influence mutually, resulting in cell apoptosis or nerve necrosis finally. Although remarkable progress has been made in the prevention and treatment of $\mathrm{CI} / \mathrm{RI}$ in the past few decades, CI/RI remains a serious global health problem, implying that it remains an unmet clinical need for developing innovative drugs for CI/RI.

$\mathrm{CHM}$ has a polypharmacological effect naturally based on the "multi-components, multi-targets and multi-pathways" principle (Guo et al., 2020; Wang et al., 2021). Based on target molecules, biological function, and bioactive compounds, current developments in network pharmacology establish an intricate interaction network that matches the natural characteristics of $\mathrm{CHM}$ and provides the detailed action mechanism of $\mathrm{CHM}$ against $\mathrm{CI} / \mathrm{RI}$ at the molecular level with a systematic viewpoint. We review senkyunolide- $\mathrm{H}$ from Conioselinum anthriscoides "Chuanxiong" [Apiaceae; Chuanxiong rhizoma], Carthamus tinctorius L. (CTL) [Asteraceae; Carthami Flos] extract/phospholipid complex, and Styrax benzoin Dryand. [Styracaceae; Benzoinum] in the paper (Zhou F. et al., 2014; Zhang Y. et al., 2018; Chen H. et al., 2020a). Furthermore, the application of various omics technologies, such as genomics and metabolomics, help investigators discover CI/RI drugs from $\mathrm{CHM}$ more comprehensively and systematically. The omics technologies are proved to be an efficient and robust way of identifying mechanisms of drug action and potential biomarkers and also afford prolific information to better understand the molecular pathogenesis of CI/RI (Liao et al., 2016; Liu S. Y. et al., 2017; Li et al., 2018; Wang R. et al., 2019). By integrating network pharmacology with metabolomics, researchers elucidate the action of CHM through constructing the "disease-targetsdrug" network and screening out the nodes of particular interest, then focusing on the alteration of endogenous metabolites in biosamples to discover the underlying mechanism of CI/RI. The strategy combines network pharmacology with omics or some advanced analysis methods and can lay a considerable foundation for explicating the effect mechanism of CHM against $\mathrm{CI} / \mathrm{RI}$ and then promoting its clinical application.

\subsection{Limitations}

Although many studies are missed in this review due to our search strategy and the limited access to some articles, the abovementioned evidence is strongly indicative that $\mathrm{CHM}$ is the emerging medicine in the prevention and/or treatment of 
CI/RI. However, some objective limitations should be considered based on the existing literature. First, many of the clinical trials are not well designed and have poor methodological quality by lacking formal inclusion/exclusion criteria, or the randomization procedures were inadequately described, the duration of therapy was too short, and conclusive results were unsatisfactory. Poorly designed and reported clinical trials usually exaggerate the treatment effects, which mislead decision making clinically. Second, CHM remedies used today have not undergone careful scientific assessment, and their preclinical studies as side effects, toxic effects, and major drug-to-drug interactions still lack in the record. Third, most of the components in CHM cannot go into the brain because they are not permeable through the BBB. The final concentration of those CHM components is very low compared with the in vitro concentration that shows some effects on cell models. Fourth, ingredients contained in one $\mathrm{CHM}$, even in the extraction of a single CHM, are very complicated. Thus, the characteristics of multicomponent, multitarget, and slow onset in CHM are not conducive to the interpretation of the action mechanism for treating CI/RI and the interaction with other medicines (including western medicines). Additionally, most previous studies on the brain protective effects of CHM are conducted in young and healthy animals, which may not simulate the prevalence of stroke in elderly patients.

\subsection{Outlooks}

Regardless, CHM deserves more attention and application. Further study should combine the holistic concept of TCM theory with modern medicine and integrate different $\mathrm{CHM}$ to achieve the optimum benefit to counter nerve injury following $\mathrm{CI} / \mathrm{IR}$ challenge. For example, a recent report suggests that the combined treatment of Ginkgo biloba dispersible tablets with nimodipine are widely used in treating CI/RI (Han et al., 2020). Furthermore, make full use of the integrated technologies, such as network pharmacology with omics or other advanced technologies to take some experimental research, searching the key part of action in CI/RI and the specific targets of CHM at a single compound level and discovering more cerebrovascular drugs from CHM. Omics technologies can characterize the molecular changes that underlie the development and progression of various complex human diseases and provide researchers with a greater understanding of the flow of information from the original cause of the disease (genetic, environmental, or developmental) to the functional consequences or relevant interactions. These technologies are proposed and heralded as the key to advancing precision medicine in the clinic (Hasin et al., 2017; Olivier et al., 2019). In the field of CI/RI, omics help reveal several key mechanisms in $\mathrm{CI} / \mathrm{RI}$ development and discover compounds from $\mathrm{CHM}$ with the bioactivities for minimizing ischemic brain injury. These compounds include sesamin, baicalin, salvianolic acid A, 6paradol, silymarin, apocynin, 3H-1,2-Dithiole-3-thione (-)-epicatechin, rutin, Dl-3-N-butylphthalide, and naringin (Chen H. et al., 2020b). Last but not least, the mechanisms of $\mathrm{CI} / \mathrm{RI}$ in humans and experimental animals cannot be equal because of species differences. This paper presents an overview on the neuroprotective effect of CHM in experimental models.
Therefore, whether CHM can exert the same protective mechanism in clinical practice requires further study. The clinical study criteria should be documented to standardize the evaluation of CHM. Currently, except for Xingnaojing injection, Naoxuekang, Xinnaoshutong, and Xuesaitong capsules (Chen et al., 2017; Lai et al., 2017), most CHM remedies used in the clinic have no ethics and dissemination registration number and trial registration number, and the trials did not follow the principles of randomization and blindness; besides this, the number of clinical samples was far from enough. These CHMs need reasonable and scientific clinical trials, and we call for more high-quality studies to confirm the neuroprotective effect and mechanism of CHM in others animal models such as dogs or rabbits in the future. In addition, international collaboration may be encouraged, promoted, and financed by the governments to improve the overall research quality. In this case, it is a promise to develop new drugs with effectiveness and safety from CHM for the prevention and treatment of patients with CI/RI.

\section{CONCLUSION}

Taken together, it cannot be ignored that CHM exerts a significant neuroprotective effect even when used alone in in vivo and in vitro studies. The results of various animal experiments further indicate that $\mathrm{CHM}$ has an intervention effect in inflammation, oxidative stress, apoptosis, $\mathrm{Ca}^{2+}$ overload, autophagy, and many others as well as the major signaling pathways in CI/RI, including PI3K/ AKT, MAPKs, NF- $\kappa$ B, Nrf2, and others. Significant progress has been made in the studies of CHM as promising drug candidates for the treatment of CI/IR. In particular, they have been gaining significant interest recently in the stroke research field given the fact that no effective neuroprotective medicine is currently available. Future studies should pay more attention to assess $\mathrm{CHM}$ that offers the best and long-term CI/RI protection as well as functional and survival rate improvement using large and different rodent animal stroke models.

\section{AUTHOR CONTRIBUTIONS}

PH drafted the manuscript. HW and CL participated in the discussion. CS and LZ prepared the figure. The corresponding author $\mathrm{YH}$ guided the formation of the entire manuscript. All authors contributed to the article and approved the submitted version.

\section{FUNDING}

This work was supported by National Science Foundation of China (No. 81873226), Zhejiang Provincial Natural Science Foundation (No. LZ18H270001), National Major Scientific and Technological Special Project for "Significant New Drugs Development" (No. 2019ZX09301101), and Zhejiang Provincial Science and Technology Innovation Leading Talent Project of “Ten Thousand Talents Plan” (2019). 


\section{REFERENCES}

An, P., Xie, J., Qiu, S., Liu, Y., Wang, J., Xiu, X., et al. (2019). Hispidulin Exhibits Neuroprotective Activities against Cerebral Ischemia Reperfusion Injury through Suppressing NLRP3-Mediated Pyroptosis. Life Sci. 232, 116599. doi:10.1016/j.lfs.2019.116599

Anisimov, A., Tvorogov, D., Alitalo, A., Leppänen, V. M., An, Y., Han, E. C., et al. (2013). Vascular Endothelial Growth Factor-Angiopoietin Chimera with Improved Properties for Therapeutic Angiogenesis. Circulation 127, 424-434. doi:10.1161/CIRCULATIONAHA.112.127472

Anrather, J., and Iadecola, C. (2016). Inflammation and Stroke: An Overview. Neurotherapeutics 13, 661-670. doi:10.1007/s13311-016-0483-x

Auriel, E., and Bornstein, N. M. (2010). Neuroprotection in Acute Ischemic StrokeCcurrent Status. J. Cel Mol Med 14, 2200-2202. doi:10.1111/j.15824934.2010.01135.X

Bai, X., Tan, T. Y., Li, Y. X., Li, Y., Chen, Y. F., Ma, R., et al. (2020). The Protective Effect of Cordyceps Sinensis Extract on Cerebral Ischemic Injury via Modulating the Mitochondrial Respiratory Chain and Inhibiting the Mitochondrial Apoptotic Pathway. Biomed. Pharmacother. 124, 109834. doi:10.1016/j.biopha.2020.109834

Barrington, J., Lemarchand, E., and Allan, S. M. (2017). A Brain in Flame; Do Inflammasomes and Pyroptosis Influence Stroke Pathology? Brain Pathol. 27, 205-212. doi:10.1111/bpa.12476

Berliocchi, L., Bano, D., and Nicotera, P. (2005). Ca2+ Signals and Death Programmes in Neurons. Philos. Trans. R. Soc. Lond. B Biol. Sci. 360, 2255-2258. doi:10.1098/rstb.2005.1765

Boehncke, W. H. (2018). Systemic Inflammation and Cardiovascular Comorbidity in Psoriasis Patients: Causes and Consequences. Front. Immunol. 9, 579. doi:10.3389/fimmu.2018.00579

Cai, M., Guo, Y., Wang, S., Wei, H., Sun, S., Zhao, G., et al. (2017). Tanshinone IIA Elicits Neuroprotective Effect through Activating the Nuclear Factor Erythroid 2-Related Factor-dependent Antioxidant Response. Rejuvenation Res. 20, 286-297. doi:10.1089/rej.2016.1912

Cai, M., Yu, Z., Wang, L., Song, X., Zhang, J., Zhang, Z., et al. (2016). Tongxinluo Reduces Brain Edema and Inhibits post-ischemic Inflammation after Middle Cerebral Artery Occlusion in Rats. J. Ethnopharmacol 181 (2), 136-145. doi:10.1016/j.jep.2016.01.026

Cao, G., Ye, X., Xu, Y., Yin, M., Chen, H., Kou, J., et al. (2016). YiQiFuMai Powder Injection Ameliorates Blood-Brain Barrier Dysfunction and Brain Edema after Focal Cerebral Ischemia-Reperfusion Injury in Mice. Drug Des. Devel Ther. 10, 315-325. doi:10.2147/DDDT.S96818

Catanese, L., Tarsia, J., and Fisher, M. (2017). Acute Ischemic Stroke Therapy Overview. Circ. Res. 120, 541-558. doi:10.1161/CIRCRESAHA.116.309278

Chang, J., Yao, X., Zou, H., Wang, L., Lu, Y., Zhang, Q., et al. (2016). BDNF/PI3K/ Akt and Nogo-A/RhoA/ROCK Signaling Pathways Contribute to Neurorestorative Effect of Houshiheisan against Cerebral Ischemia Injury in Rats. J. Ethnopharmacol 194 (24), 1032-1042. doi:10.1016/j.jep.2016.11.005

Chang, L., Yin, C. Y., Wu, H. Y., Tian, B. B., Zhu, Y., Luo, C. X., et al. (2017). (+)-Borneol Is Neuroprotective against Permanent Cerebral Ischemia in Rats by Suppressing Production of Proinflammatory Cytokines. J. Biomed. Res. 31, 306-314. doi:10.7555/JBR.31.20160138

Chang, L. L., Li, C., Li, Z. L., Wei, Z. L., Jia, X. B., Pang, S. T., et al. (2020). Carthamus tinctorius L. Extract Ameliorates Cerebral Ischemia-Reperfusion Injury in Rats by Regulating Matrix Metalloproteinases and Apoptosis. Indian J. Pharmacol. 52 (2), 108-116. Epub 2020 Jun 3. PMID: 32565598; PMCID: PMC7282686. doi:10.4103/ijp.IJP_400_18

Chen, A. L. (2016). Protective Effect of Mu-Xiang-You-Fang on PC12 Cell Injury Induced by Oxygen-Glucose Deprivation and Reperfusion Based on AMPK/ mTOR Signaling Pathway. Ningxia, China: Master's Degree of Ningxia Medical University.

Chen, H., Cao, H., Guo, X., Zhao, M., Xia, Q., Chen, B., et al. (2017). Naoxuekang, Xinnaoshutong and Xuesaitong capsules for treating stroke: a protocol for a randomised controlled trial. BMJ Open 7(11), e015983. doi:10.1136/bmjopen2017-015983

Chen, H., He, Y., Chen, S., Qi, S., and Shen, J. (2020b). Therapeutic Targets of Oxidative/nitrosative Stress and Neuroinflammation in Ischemic Stroke:
Applications for Natural Product Efficacy with Omics and Systemic Biology. Pharmacol. Res. 158, 104877. doi:10.1016/j.phrs.2020.104877

Chen, H., Ren, M., Li, H., Xie, Q., Ma, R., Li, Y., et al. (2020a). Neuroprotection of Benzoinum in Cerebral Ischemia Model Rats via the ACE-AngI-VEGF Pathway. Life Sci. 260, 118418. doi:10.1016/j.lfs.2020.118418

Chen, K. Y., Wu, K. C., Hueng, D. Y., Huang, K. F., and Pang, C. Y. (2020). Antiinflammatory Effects of Powdered Product of Bu Yang Huan Wu Decoction: Possible Role in Protecting against Transient Focal Cerebral Ischemia. Int. J. Med. Sci. 17 (12), 1854-1863. doi:10.7150/ijms.46581

Chen, L., Wang, X., Zhang, J., Dang, C., Liu, G., Liang, Z., et al. (2016). Tongxinluo Enhances Neurogenesis and Angiogenesis in Peri-Infarct Area and Subventricular Zone and Promotes Functional Recovery after Focal Cerebral Ischemic Infarction in Hypertensive Rats. Evid. Based Complement. Alternat Med. 2016, 8549590. doi:10.1155/2016/8549590

Chen, L., Xiang, Y., Kong, L., Zhang, X., Sun, B., Wei, X., et al. (2013). Hydroxysafflor Yellow A Protects against Cerebral Ischemia-Reperfusion Injury by Anti-apoptotic Effect through PI3K/Akt/GSK3 $\beta$ Pathway in Rat. Neurochem. Res. 38 (11), 2268-2275. doi:10.1007/s11064-013-1135-8

Chen, L., Zhao, Y., Zhang, T., Dang, X., Xie, R., Li, Z., et al. (2014). Protective Effect of Sheng-Nao-Kang Decoction on Focal Cerebral Ischemia-Reperfusion Injury in Rats. J. Ethnopharmacol 151, 228-236. doi:10.1016/j.jep.2013.10.015

Chen, S., Peng, H., Rowat, A., Gao, F., Zhang, Z., Wang, P., et al. (2015). The Effect of Concentration and Duration of Normobaric Oxygen in Reducing Caspase-3 and -9 Expression in a Rat-Model of Focal Cerebral Ischaemia. Brain Res. 1618, 205-211. doi:10.1016/j.brainres.2015.05.027

Chen X, X., Wu, H., Chen, H., Wang, Q., Xie, X. J., and Shen, J. (2019). Astragaloside VI Promotes Neural Stem Cell Proliferation and Enhances Neurological Function Recovery in Transient Cerebral Ischemic Injury via Activating EGFR/MAPK Signaling Cascades. Mol. Neurobiol. 56, 3053-3067. doi:10.1007/s12035-018-1294-3

Chen Z. Z, Z.-Z., Gong, X., Guo, Q., Zhao, H., and Wang, L. (2019). Bu Yang Huan Wu Decoction Prevents Reperfusion Injury Following Ischemic Stroke in Rats via Inhibition of HIF-1 $\alpha$, VEGF and Promotion $\beta$-ENaC Expression. J. Ethnopharmacology 228, 70-81. doi:10.1016/j.jep.2018.09.017

Cheng, Q., Tong, F., Shen, Y., He, C., Wang, C., and Ding, F. (2019). Achyranthes Bidentata Polypeptide K Improves Long-Term Neurological Outcomes through Reducing Downstream Microvascular Thrombosis in Experimental Ischemic Stroke. Brain Res. 1706, 166-176. doi:10.1016/j.brainres.2018.11.010

Cheng, X., Hou, Z., Sun, J., Huang, Y., Wang, L., Zhou, Z., et al. (2017). Protective Effects of Tongxinluo on Cerebral Ischemia/reperfusion Injury Related to Connexin 43/Calpain II/Bax/Caspase-3 Pathway in Rat. J. Ethnopharmacol 198, 148-157. doi:10.1016/j.jep.2017.01.004

Cheng, X., Luo, H., Zhou, L., Wang, L., Sun, J., Huang, Y., et al. (2014). Neuroprotective Effect of the Traditional Chinese Herbal Formula Tongxinluo: a PET Imaging Study in Rats. Neural Regen. Res. 9 (13), 1267-1274. doi:10.4103/1673-5374.137573

Dang, X., Miao, J. J., Chen, A. Q., Li, P., Chen, L., Liang, J. R., et al. (2015). The Antithrombotic Effect of RSNK in Blood-Stasis Model Rats. J. Ethnopharmacol 173 (173), 266-272. doi:10.1016/j.jep.2015.06.030

Dawson, T. M., and Dawson, V. L. (2017). Mitochondrial Mechanisms of Neuronal Cell Death: Potential Therapeutics. Annu. Rev. Pharmacol. Toxicol. 57, 437-454. doi:10.1146/annurev-pharmtox-010716-105001

Dong, X., Gao, J., Zhang, C. Y., Hayworth, C., Frank, M., and Wang, Z. (2019). Neutrophil Membrane-Derived Nanovesicles Alleviate Inflammation to Protect Mouse Brain Injury from Ischemic Stroke. ACS Nano 13, 1272-1283. doi:10.1021/acsnano.8b06572

Du, C. P., Tan, R., and Hou, X. Y. (2012). Fyn Kinases Play a Critical Role in Neuronal Apoptosis Induced by Oxygen and Glucose Deprivation or Amyloid$\beta$ Peptide Treatment. CNS Neurosci. Ther. 18, 754-761. doi:10.1111/j.17555949.2012.00357.x

Fan, R. J., Luo, Y. F., Chen, Y. S., He, G. Z., Tang, Z. S., and Lu, Y. (2015). The Effects of Taohong Siwu Tang on the Caspase-3 and P53 Expression in Cerebral Cortex Neurons of Rat Cerebral Ischemia Reperfusion Injury Model. Chin. J. Neur 31 (06), 739-745. doi:10.16557/j.cnki.1000-7547.2015.06.0012

Fang, X., Li, Y., Qiao, J., Guo, Y., and Miao, M. (2017). Neuroprotective Effect of Total Flavonoids from Ilex Pubescens against Focal Cerebral Ischemia/reperfusion Injury in Rats. Mol. Med. Rep. 16, 7439-7449. doi:10.3892/mmr.2017.7540 
Feng, C., Wan, H., Zhang, Y., Yu, L., Shao, C., He, Y., et al. (2020). Neuroprotective Effect of Danhong Injection on Cerebral Ischemia-Reperfusion Injury in Rats by Activation of the PI3K-Akt Pathway. Front. Pharmacol. 11, 298. doi:10.3389/ fphar.2020.00298

Fu, P. K., Pan, T. L., Yang, C. Y., Jeng, K. C., Tang, N. Y., and Hsieh, C. L. (2016). Carthamus tinctorius L. Ameliorates Brain Injury Followed by Cerebral Ischemia-Reperfusion in Rats by Antioxidative and Anti-inflammatory Mechanisms. Iran J. Basic Med. Sci. 19, 1368-1375. doi:10.22038/ ijbms.2016.7925

Fu, X., Wang, J., Liao, S., Lv, Y., Xu, D., Yang, M., et al. (2019). 1H NMR-Based Metabolomics Reveals Refined-Huang-Lian-Jie-Du-Decoction (BBG) as a Potential Ischemic Stroke Treatment Drug with Efficacy and a Favorable Therapeutic Window. Front. Pharmacol. 10, 337. doi:10.3389/fphar.2019.00337

Geng, X., Parmar, S., Li, X., Peng, C., Ji, X., Chakraborty, T., et al. (2013). Reduced Apoptosis by Combining Normobaric Oxygenation with Ethanol in Transient Ischemic Stroke. Brain Res. 1531, 17-24. doi:10.1016/j.brainres.2013.07.051

Guan, X., Li, Z., Zhu, S., Cheng, M., Ju, Y., Ren, L., et al. (2021). Galangin Attenuated Cerebral Ischemia-Reperfusion Injury by Inhibition of Ferroptosis through Activating the SLC7A11/GPX4 axis in Gerbils. Life Sci. 264, 118660. doi:10.1016/j.lfs.2020.118660

Guo, F., Tang, X., Zhang, W., Wei, J., Tang, S., Wu, H., et al. (2020). Exploration of the Mechanism of Traditional Chinese Medicine by AI Approach Using Unsupervised Machine Learning for Cellular Functional Similarity of Compounds in Heterogeneous Networks, XiaoErFuPi Granules as an Example. Pharmacol. Res. 160, 105077. doi:10.1016/j.phrs.2020.105077

Guo, H., Li, M. J., Liu, Q. Q., Guo, L. L., Ma, M. M., Wang, S. X., et al. (2014). Danhong Injection Attenuates Ischemia/reperfusion-Induced Brain Damage Which Is Associating with Nrf2 Levels In Vivo and In Vitro. Neurochem. Res. 39 (9), 1817-1824. doi:10.1007/s11064-014-1384-1

Guo, R. B., Wang, G. F., Zhao, A. P., Gu, J., Sun, X. L., and Hu, G. (2012). Paeoniflorin Protects against Ischemia-Induced Brain Damages in Rats via Inhibiting MAPKs/NF-Kb-Mediated Inflammatory Responses. PLoS One 7, e49701. doi:10.1371/journal.pone.0049701

Guo, Y., Yan, S., Xu, L., Zhu, G., Yu, X., and Tong, X. (2014). Use of Angong Niuhuang in Treating central Nervous System Diseases and Related Research. Evid. Based Complement. Alternat Med. 2014, 346918. doi:10.1155/2014/346918

Han, J., Xiao, Q., Lin, Y. H., Zheng, Z. Z., He, Z. D., Hu, J., et al. (2015). Neuroprotective Effects of Salidroside on Focal Cerebral Ischemia/ reperfusion Injury Involve the Nuclear Erythroid 2-related Factor 2 Pathway. Neural Regen. Res. 10, 1989-1996. doi:10.4103/1673-5374.172317

Han, J., Xu, H. H., Chen, X. L., Hu, H. R., Hu, K. M., Chen, Z. W., et al. (2018). Total Flavone of Rhododendron Improves Cerebral Ischemia Injury by Activating Vascular TRPV4 to Induce Endothelium-Derived Hyperpolarizing FactorMediated Responses. Evid. Based Complement. Alternat Med. 2018, 8919867. doi:10.1155/2018/8919867

Han, K., Rong, W., Wang, Q., Qu, J., Li, Q., Bi, K., et al. (2020). Time-dependent Metabolomics Study of Cerebral Ischemia-Reperfusion and its Treatment: Focus on the Combination of Traditional Chinese Medicine and Western Medicine. Anal. Bioanal. Chem. 412 (26), 7195-7209. doi:10.1007/s00216-020-02852-w

Hasin, Y., Seldin, M., and Lusis, A. (2017). Multi-omics Approaches to Disease. Genome Biol. 18 (1), 83. doi:10.1186/s13059-017-1215-1

He, Q., Sun, J., Wang, Q., Wang, W., and He, B. (2014). Neuroprotective Effects of Ginsenoside Rg1 against Oxygen-Glucose Deprivation in Cultured Hippocampal Neurons. J. Chin. Med. Assoc. 77, 142-149. doi:10.1016/ j.jcma.2014.01.001

Hou, K., Xu, D., Li, F., Chen, S., and Li, Y. (2019). The Progress of Neuronal Autophagy in Cerebral Ischemia Stroke: Mechanisms, Roles and Research Methods. J. Neurol. Sci. 400, 72-82. doi:10.1016/j.jns.2019.03.015

Hu, G. Q., Du, X., Li, Y. J., Gao, X. Q., Chen, B. Q., and Yu, L. (2017). Inhibition of Cerebral Ischemia/reperfusion Injury-Induced Apoptosis: Nicotiflorin and JAK2/ STAT3 Pathway. Neural Regen. Res. 12 (1), 96-102. doi:10.4103/1673-5374.198992

Hu, H. X., Lin, R. H., Zhu, X. Q., Li, Z. F., and Chen, L. D. (2015). Antiinflammatory Effects of Gualou Guizhi Decoction in Transient Focal Cerebral Ischemic Brains. [Corrected]. Mol. Med. Rep. 12 (1), 1321-1327. doi:10.3892/mmr.2015.3511

Hu, J., Pang, W. S., Han, J., Zhang, K., Zhang, J. Z., and Chen, L. D. (2018). Gualou Guizhi Decoction Reverses Brain Damage with Cerebral Ischemic Stroke, Multi-Component Directed Multi-Target to Screen Calcium-Overload
Inhibitors Using Combination of Molecular Docking and Protein-Protein Docking. J. Enzyme Inhib. Med. Chem. 33, 115-125. doi:10.1080/ 14756366.2017.1396457

Huang, S., Gong, T., Zhang, T., Wang, X., Cheng, Q., and Li, Y. (2019). Zhongfenggao Protects Brain Microvascular Endothelial Cells from Oxygen-Glucose Deprivation/Reoxygenation-Induced Injury by Angiogenesis. Biol. Pharm. Bull. 42, 222-230. doi:10.1248/bpb.b18-00650

Ji, K., Xue, L., Cheng, J., and Bai, Y. (2016). Preconditioning of H2S Inhalation Protects against Cerebral Ischemia/reperfusion Injury by Induction of HSP70 through PI3K/Akt/Nrf2 Pathway. Brain Res. Bull. 121, 68-74. doi:10.1016/ j.brainresbull.2015.12.007

Jia, Y., Zuo, D., Li, Z., Liu, H., Dai, Z., Cai, J., et al. (2014). Astragaloside IV Inhibits Doxorubicin-Induced Cardiomyocyte Apoptosis Mediated by Mitochondrial Apoptotic Pathway via Activating the PI3K/Akt Pathway. Chem. Pharm. Bull. (Tokyo) 62, 45-53. doi:10.1248/cpb.c13-00556

Jiang, Y., and Lian, Y. J. (2015). Effects of Danhong Injection on Hemodynamics and the Inflammation-Related NF-Kb Signaling Pathway in Patients with Acute Cerebral Infarction. Genet. Mol. Res. 14 (4), 16929-16937. doi:10.4238/2015

Jiang, Y. F., Liu, Z. Q., Cui, W., Zhang, W. T., Gong, J. P., Wang, X. M., et al. (2015). Antioxidant Effect of Salvianolic Acid B on Hippocampal CA1 Neurons in Mice with Cerebral Ischemia and Reperfusion Injury. Chin. J. Integr. Med. 21 (7), 516-522. doi:10.1007/s11655-014-1791-1

Kim, J. Y., Kawabori, M., and Yenari, M. A. (2014). Innate Inflammatory Responses in Stroke: Mechanisms and Potential Therapeutic Targets. Curr. Med. Chem. 21, 2076-2097. doi:10.2174/0929867321666131228205146

Kopáni, M., Celec, P., Danisovic, L., Michalka, P., and Biró, C. (2006). Oxidative Stress and Electron Spin Resonance. Clin. Chim. Acta 364, 61-66. doi:10.1016/ j.cca.2005.05.016

Kovacs, S. B., and Miao, E. A. (2017). Gasdermins: Effectors of Pyroptosis. Trends Cel Biol 27, 673-684. doi:10.1016/j.tcb.2017.05.005

Kyriakis, J. M., and Avruch, J. (2012). Mammalian Mapk Signal Transduction Pathways Activated by Stress and Inflammation: A 10-Year Update. Physiol. Rev. 92, 689-737. doi:10.1152/physrev.00028.2011

Lai, X., Cao, K., Kong, L., Liu, Q., and Gao, Y. (2017). XMAS study investigators. Xingnaojing for Moderate-to-severe Acute ischemic Stroke (XMAS): study protocol for a randomized controlled trial. Trials 18(1), 478-124. doi:10.1186/ s13063-017-2222-y

Lambertsen, K. L., Finsen, B., and Clausen, B. H. (2019). Post-stroke Inflammation-Target or Tool for Therapy? Acta Neuropathol. 137, 693-714. doi:10.1007/s00401-018-1930-z

Lan, B., Ge, J. W., Cheng, S. W., Zheng, X. L., Liao, J., He, C., et al. (2020). Extract of Naotaifang, a Compound Chinese Herbal Medicine, Protects Neuron Ferroptosis Induced by Acute Cerebral Ischemia in Rats. J. Integr. Med. 18, 344-350. doi:10.1016/j.joim.2020.01.008

Lei, P., Bai, T., and Sun, Y. (2019). Mechanisms of Ferroptosis and Relations with Regulated Cell Death: A Review. Front. Physiol. 10, 139. doi:10.3389/ fphys.2019.00139

Li, F., Li, W., Li, X., Li, F., Zhang, L., Wang, B., et al. (2016). Geniposide Attenuates Inflammatory Response by Suppressing P2Y14 Receptor and Downstream ERK1/2 Signaling Pathway in Oxygen and Glucose Deprivation-Induced Brain Microvascular Endothelial Cells. J. Ethnopharmacol 185, 77-86. doi:10.1016/j.jep.2016.03.025

Li, J., Cao, F., Yin, H. L., Huang, Z. J., Lin, Z. T., Mao, N., et al. (2020). Ferroptosis: Past, Present and Future. Cell Death Dis 11, 88. doi:10.1038/s41419-020-2298-2

Li, L., Zhang, X., Cui, L., Wang, L., Liu, H., Ji, H., et al. (2013). Ursolic Acid Promotes the Neuroprotection by Activating Nrf2 Pathway after Cerebral Ischemia in Mice. Brain Res. 1497, 32-39. doi:10.1016/j.brainres.2012.12.032

Li, L. M., Zheng, B., Zhang, R. N., Jin, L. S., Zheng, C. Y., Wang, C., et al. (2015). Chinese Medicine Tongxinluo Increases Tight junction Protein Levels by Inducing KLF5 Expression in Microvascular Endothelial Cells. Cell Biochem Funct 33 (4), 226-234. doi:10.1002/cbf.3108

Li, M. H., Ruan, L. Y., Chen, C., Xing, Y. X., Hong, W., Du, R. H., et al. (2018). Protective Effects of Polygonum Multiflorum on Ischemic Stroke Rat Model Analysed by $1 \mathrm{H}$ NMR Metabolic Profiling. J. Pharm. Biomed. Anal. 155, 91-103. doi:10.1016/j.jpba.2018.03.049

Li, W. H., Yang, Y. L., Cheng, X., Liu, M., Zhang, S. S., Wang, Y. H., et al. (2020). Baicalein Attenuates Caspase-independent Cells Death via Inhibiting PARP-1 Activation and AIF Nuclear Translocation in Cerebral Ischemia/reperfusion Rats. Apoptosis 25, 354-369. doi:10.1007/s10495-020-01600-w 
Li, X., Huang, L., Liu, G., Fan, W., Li, B., Liu, R., et al. (2020). Ginkgo Diterpene Lactones Inhibit Cerebral Ischemia/reperfusion Induced Inflammatory Response in Astrocytes via TLR4/NF-Kb Pathway in Rats. J. Ethnopharmacol 249, 112365. doi:10.1016/j.jep.2019.112365

Li, Y., Ren, M., Wang, J., Ma, R., Chen, H., Xie, Q., et al. (2021). Progress in Borneol Intervention for Ischemic Stroke: A Systematic Review. Front. Pharmacol. 12, 606682. doi:10.3389/fphar.2021.606682

Li, Y., Yang, Y., Zhao, Y., Zhang, J., Liu, B., Jiao, S., et al. (2019). Astragaloside IV Reduces Neuronal Apoptosis and Parthanatos in Ischemic Injury by Preserving Mitochondrial Hexokinase-II. Free Radic. Biol. Med. 131, 251-263. doi:10.1016/ j.freeradbiomed.2018.11.033

Liao, J., Wei, B., Chen, H., Liu, Y., and Wang, J. (2016). Bioinformatics Investigation of Therapeutic Mechanisms of Xuesaitong Capsule Treating Ischemic Cerebrovascular Rat Model with Comparative Transcriptome Analysis. Am. J. Transl Res. 8, 2438-2449. Available at: https://www.ncbi. nlm.nih.gov/pubmed/27347353.

Liao, J., Xia, X., Wang, G. Z., Shi, Y. M., and Ge, J. W. (2015). Naotaifang Extract Treatment Results in Increased Ferroportin Expression in the hippocampus of Rats Subjected to Cerebral Ischemia. Mol. Med. Rep. 11, 4047-4052. doi:10.3892/mmr.2015.3309

Liu, B., Li, F., Shi, J., Yang, D., Deng, Y., and Gong, Q. (2016). Gastrodin Ameliorates Subacute Phase Cerebral Ischemia-reperfusion I-njury by I-nhibiting I-nflammation and A-poptosis in R-ats. Mol. Med. Rep. 14 (5), 4144-4152. doi: $10.3892 / \mathrm{mmr} .2016 .5785$

Liu, D., Dong, Z., Xiang, F., Liu, H., Wang, Y., Wang, Q., et al. (2020). Dendrobium Alkaloids Promote Neural Function after Cerebral Ischemia-Reperfusion Injury through Inhibiting Pyroptosis Induced Neuronal Death in Both In Vivo and In Vitro Models. Neurochem. Res. 45, 437-454. doi:10.1007/ s11064-019-02935-w

Liu, H., Yan, Y., Pang, P., Mao, J., Hu, X., Li, D., et al. (2019). Angong Niuhuang Pill as Adjuvant Therapy for Treating Acute Cerebral Infarction and Intracerebral Hemorrhage: A Meta-Analysis of Randomized Controlled Trials. J. Ethnopharmacol 237 (237), 307-313. doi:10.1016/j.jep.2019.03.043

Liu, R., Yu, X., Zhang, L., Zhang, H., Gong, Y., Wu, K., et al. (2020). Computed Tomography (CT) Imaging Evaluation of Integrated Traditional Chinese Medicine Cooperative Therapy in Treating Acute Cerebral Infarction: A Randomized Controlled Trial. Medicine (Baltimore) 99, e19998. doi:10.1097/ MD.0000000000019998

Liu, S. Y., Cai, W., Wang, F., Liu, Y., Shang, Z. P., Zhang, X. P., et al. (2017). UHPLC-LTQ-Orbitrap-based Metabolomics Coupled with Metabolomics Pathway Analysis Method for Exploring the protection Mechanism of Kudiezi Injection in a Rat Anti-ischemic Cerebral Reperfusion Damage Model. Chin. J. Nat. Med. 15, 955-960. doi:10.1016/S1875-5364(18)30013-X

Liu, X., Chen, X., Zhu, Y., Wang, K., and Wang, Y. (2017). Effect of Magnolol on Cerebral Injury and Blood Brain Barrier Dysfunction Induced by IschemiaReperfusion In Vivo and In Vitro. Metab. Brain Dis. 32, 1109-1118. doi:10.1007/s11011-017-0004-6

Liu, X. (2017). Network Pharmacology-Based Study on Action Mechanism of Ypf Power Treating for Asthma. Chengdu, China: Southwest Jiaotong University.

Liu, Y., Gao, J., Peng, M., Meng, H., Ma, H., Cai, P., et al. (2018). A Review on Central Nervous System Effects of Gastrodin. Front. Pharmacol. 9, 24. doi:10.3389/fphar.2018.00024

Liu, Y., Tang, Q., Shao, S., Chen, Y., Chen, W., and Xu, X. (2017). Lyophilized Powder of Catalpol and Puerarin Protected Cerebral Vessels from Ischemia by its Anti-apoptosis on Endothelial Cells. Int. J. Biol. Sci. 13, 327-338. doi:10.7150/ijbs.17751

Liu, Z., Gan, L., Xu, Y., Luo, D., Ren, Q., Wu, S., et al. (2017). Melatonin Alleviates Inflammasome-Induced Pyroptosis through Inhibiting NF-Kb/GSDMD Signal in Mice Adipose Tissue. J. Pineal Res. 63, 63. doi:10.1111/jpi.12414

Long, J., Gao, M., Kong, Y., Shen, X., Du, X., Son, Y. O., et al. (2012). Cardioprotective Effect of Total Paeony Glycosides against IsoprenalineInduced Myocardial Ischemia in Rats. Phytomedicine 19, 672-676. doi:10.1016/j.phymed.2012.03.004

Ma, C., Wang, X., Xu, T., Yu, X., Zhang, S., Liu, S., et al. (2019). Qingkailing Injection Ameliorates Cerebral Ischemia-Reperfusion Injury and Modulates the AMPK/NLRP3 Inflammasome Signalling Pathway. BMC Complement. Altern. Med. 19, 320. doi:10.1186/s12906-019-2703-5
Ma, C., Wang, X., Xu, T., Zhang, S., Liu, S., Zhai, C., et al. (2020). An Integrative Pharmacology-Based Analysis of Refined Qingkailing Injection against Cerebral Ischemic Stroke: A Novel Combination of Baicalin, Geniposide, Cholic Acid, and Hyodeoxycholic Acid. Front. Pharmacol. 11 (11), 519. doi:10.3389/fphar.2020.00519

Ma, Y., Wang, W., Yang, J., Zhang, S., Li, Z., Li, F., et al. (2020). A Network Pharmacology Technique to Investigate the Synergistic Mechanisms of Salvia Miltiorrhiza and Radix Puerariae in Treatment of Cardio-Cerebral Vascular Diseases. Evid. Based Complement. Alternat Med. 2020, 6937186. doi:10.1155/ $2020 / 6937186$

Manwani, B., and McCullough, L. D. (2013). Function of the Master Energy Regulator Adenosine Monophosphate-Activated Protein Kinase in Stroke. J. Neurosci. Res. 91, 1018-1029. doi:10.1002/jnr.23207

Maugeri, R., Schiera, G., Di Liegro, C. M., Fricano, A., Iacopino, D. G., and Di Liegro, I. (2016). Aquaporins and Brain Tumors. Int. J. Mol. Sci. 17, 17. doi:10.3390/ijms17071029

Mei, Z. G., Tan, L. J., Wang, J. F., Li, X. L., Huang, W. F., and Zhou, H. J. (2017). Fermented Chinese Formula Shuan-Tong-Ling Attenuates Ischemic Stroke by Inhibiting Inflammation and Apoptosis. Neural Regen. Res. 12, 425-432. doi:10.4103/1673-5374.202946

Meng, H., Jin, W., Yu, L., Xu, S., Wan, H., and He, Y. (2021). Protective Effects of Polysaccharides on Cerebral Ischemia: A Mini-Review of the Mechanisms. Int. J. Biol. Macromol 169, 463-472. doi:10.1016/j.ijbiomac10.1016/ j.ijbiomac.2020.12.124

Miao, M. S., Guo, L., Li, R. Q., and Zhang, X. L. (2016). Radix Ilicis Pubescentis Total Flavonoids Ameliorates Neuronal Damage and Reduces Lesion Extent in a Mouse Model of Transient Ischemic Attack. Neural Regen. Res. 11, 441-446. doi:10.4103/1673-5374.179056

Mizushima, N., and Komatsu, M. (2011). Autophagy: Renovation of Cells and Tissues. Cell 147, 728-741. doi:10.1016/j.cell.2011.10.026

Mizushima, N. (2010). The Role of the Atg1/ULK1 Complex in Autophagy Regulation. Curr. Opin. Cel Biol 22, 132-139. doi:10.1016/j.ceb.2009.12.004

Moritz, M., Pfeifer, S., Balmayor, E. R., Mittermayr, R., Wolbank, S., Redl, H., et al. (2017). VEGF Released from a Fibrin Biomatrix Increases VEGFR-2 Expression and Improves Early Outcome after Ischaemia-Reperfusion Injury. J. Tissue Eng. Regen. Med. 11, 2153-2163. doi:10.1002/term.2114

Nan, L., Xie, Q., Chen, Z., Zhang, Y., Chen, Y., Li, H., et al. (2020). Involvement of PARP-1/AIF Signaling Pathway in Protective Effects of Gualou Guizhi Decoction against Ischemia-Reperfusion Injury-Induced Apoptosis. Neurochem. Res. 45 (2), 278-294. doi:10.1007/s11064-019-02912-3

Navarro-Yepes, J., Zavala-Flores, L., Anandhan, A., Wang, F., Skotak, M., Chandra, N., et al. (2014). Antioxidant Gene Therapy against Neuronal Cell Death. Pharmacol. Ther. 142, 206-230. doi:10.1016/j.pharmthera.2013.12.007

Nederlof, R., Eerbeek, O., Hollmann, M. W., Southworth, R., and Zuurbier, C. J. (2014). Targeting Hexokinase II to Mitochondria to Modulate Energy Metabolism and Reduce Ischaemia-Reperfusion Injury in Heart. $\mathrm{Br}$. J. Pharmacol. 171, 2067-2079. doi:10.1111/bph.12363

O'Connell, G. C., Chantler, P. D., and Barr, T. L. (2017). Stroke-associated Pattern of Gene Expression Previously Identified by Machine-Learning Is Diagnostically Robust in an Independent Patient Population. Genom Data 14, 47-52. doi:10.1016/j.gdata.2017.08.006

Olivier, M., Asmis, R., Hawkins, G. A., Howard, T. D., and Cox, L. A. (2019). The Need for Multi-Omics Biomarker Signatures in Precision Medicine. Int. J. Mol. Sci. 20 (19), 4781. doi:10.3390/ijms20194781

Olmez, I., and Ozyurt, H. (2012). Reactive Oxygen Species and Ischemic Cerebrovascular Disease. Neurochem. Int. 60, 208-212. doi:10.1016/ j.neuint.2011.11.009

Ong, W. Y., Farooqui, T., Koh, H. L., Farooqui, A. A., and Ling, E. A. (2015). Protective Effects of Ginseng on Neurological Disorders. Front. Aging Neurosci. 7, 129-142. doi:10.3389/fnagi.2015.00129

Orgah, J. O., Ren, J., Liu, X., Orgah, E. A., Gao, X. M., and Zhu, Y. (2019). Danhong Injection Facilitates Recovery of post-stroke Motion Deficit via ParkinEnhanced Mitochondrial Function. Restor Neurol. Neurosci. 37 (4), 375-395. doi:10.3233/RNN-180828

Orrenius, S., Zhivotovsky, B., and Nicotera, P. (2003). Regulation of Cell Death: the Calcium-Apoptosis Link. Nat. Rev. Mol. Cel Biol 4, 552-565. doi:10.1038/ nrm1150 
Pan, R., Cai, J., Zhan, L., Guo, Y., Huang, R. Y., Li, X., et al. (2017). Buyang Huanwu Decoction Facilitates Neurorehabilitation through an Improvement of Synaptic Plasticity in Cerebral Ischemic Rats. BMC Complement. Altern. Med. 17 (1), 173. doi:10.1186/s12906-017-1680-9

Pan, X. W., Wang, M. J., Gong, S. S., Sun, M. H., Wang, Y., Zhang, Y. Y., et al. (2020). YiQiFuMai Lyophilized Injection Ameliorates tPA-Induced Hemorrhagic Transformation by Inhibiting Cytoskeletal Rearrangement Associated with ROCK1 and NF-kappaB Signaling Pathways. J. Ethnopharmacol 262, 113161. doi:10.1016/j.jep.2020.113161

Peng, Z., Wang, S., Chen, G., Cai, M., Liu, R., Deng, J., et al. (2015). Gastrodin Alleviates Cerebral Ischemic Damage in Mice by Improving Anti-oxidant and Anti-inflammation Activities and Inhibiting Apoptosis Pathway. Neurochem. Res. 40, 661-673. doi:10.1007/s11064-015-1513-5

Pereira, E. R., Frudd, K., Awad, W., and Hendershot, L. M. (2014). Endoplasmic Reticulum (ER) Stress and Hypoxia Response Pathways Interact to Potentiate Hypoxia-Inducible Factor 1 (HIF-1) Transcriptional Activity on Targets like Vascular Endothelial Growth Factor (VEGF). J. Biol. Chem. 289, 3352-3364. doi:10.1074/jbc.M113.507194

Qiu, Y., Cao, Y., Cao, W., Jia, Y., and Lu, N. (2020). The Application of Ferroptosis in Diseases. Pharmacol. Res. 159, 104919. doi:10.1016/j.phrs.2020.104919

Rai, A. T., Seldon, A. E., Boo, S., Link, P. S., Domico, J. R., and Tarabishy, A. R. (2016). A Population-Based Incidence of Acute Large Vessel Occlusions and Thrombectomy Eligible Patients Indicates Significant Potential for Growth of Endovascular Stroke Therapy in the USA. J. Neurointerv Surg. 9 (8), 722-726. doi:10.1136/neurintsurg-2016-012515

Robinson, N., Ganesan, R., Hegedus, C., Kovacs, K., Kufer, T. A., and Virag, L. (2019). Programmed Necrotic Cell Death of Macrophages: Focus on Pyroptosis, Necroptosis, and Parthanatos. Redox Biol. 26, 101239. doi:10.1016/ j.redox.2019.101239

Santa-Cecilia, F. V., Socias, B., Ouidja, M. O., Sepulveda-Diaz, J. E., Acuna, L., Silva, R. L., et al. (2016). Doxycycline Suppresses Microglial Activation by Inhibiting the P38 MAPK and NF-kB Signaling Pathways. Neurotox Res. 29, 447-459. doi:10.1007/s12640-015-9592-2

Shen, J., Huang, K., Zhu, Y., Xu, K., Zhan, R., and Pan, J. (2020). Buyang Huanwu Decoction Promotes Angiogenesis after Cerebral Ischemia by Inhibiting the Nox4/ROS Pathway. Evid. Based Complement. Alternat Med. 2020, 5264205. doi:10.1155/2020/5264205

Shi, A., Xiang, J., He, F., Zhu, Y., Zhu, G., Lin, Y., et al. (2018). The Phenolic Components of Gastrodia Elata Improve Prognosis in Rats after Cerebral Ischemia/Reperfusion by Enhancing the Endogenous Antioxidant Mechanisms. Oxid Med. Cel Longev 2018, 7642158. doi:10.1155/2018/7642158

Shi, J., Zhao, Y., Wang, K., Shi, X., Wang, Y., Huang, H., et al. (2015). Cleavage of GSDMD by Inflammatory Caspases Determines Pyroptotic Cell Death. Nature 526, 660-665. doi:10.1038/nature15514

Son, T. G., Camandola, S., Arumugam, T. V., Cutler, R. G., Telljohann, R. S., Mughal, M. R., et al. (2010). Plumbagin, a Novel Nrf2/ARE Activator, Protects against Cerebral Ischemia. J. Neurochem. 112, 1316-1326. doi:10.1111/j.14714159.2009.06552.x

Sonkusare, S. K., Bonev, A. D., Ledoux, J., Liedtke, W., Kotlikoff, M. I., Heppner, T. J., et al. (2012). Elementary Ca2+ Signals through Endothelial TRPV4 Channels Regulate Vascular Function. Science 336, 597-601. doi:10.1126/science.1216283

Subedi, L., and Gaire, B. P. (2021). Phytochemicals as Regulators of Microglia/ macrophages Activation in Cerebral Ischemia. Pharmacol. Res. 165, 105419. doi:10.1016/j.phrs.2021.105419

Sun, C., Lai, X., Huang, X., and Zeng, Y. (2014). Protective Effects of Ginsenoside Rg1 on Astrocytes and Cerebral Ischemic-Reperfusion Mice. Biol. Pharm. Bull. 37 (12), 1891-1898. doi:10.1248/bpb.b14-00394

Sun, Y. J., Zhang, N. N., and Zhang, M. (2019). Effect of Resveratrol on Cellular Pyrolysisof Rat Brain during Ischemia Reperfusion and on NLRP3 Inflammatory Bodies, Caspase-1 and ZO-1 in Microglia. J. Hainan Med. Univ. 25 (17), 1291-1294. doi:10.13210/j.cnki.jhmu.20190716.002

Szarka, N., Pabbidi, M. R., Amrein, K., Czeiter, E., Berta, G., Pohoczky, K., et al. (2018). Traumatic Brain Injury Impairs Myogenic Constriction of Cerebral Arteries: Role of Mitochondria-Derived H2O2 and TRPV4-dependent Activation of BKca Channels. J. Neurotrauma 35, 930-939. doi:10.1089/ neu.2017.5056

Tan, L., Wang, Y., Jiang, Y., Wang, R., Zu, J., and Tan, R. (2020). Hydroxysafflor Yellow A Together with Blood-Brain Barrier Regulator Lexiscan for Cerebral
Ischemia Reperfusion Injury Treatment. ACS Omega 5, 19151-19164. doi:10.1021/acsomega.0c02502

Tsoi, B., Chen, X., Gao, C., Wang, S., Yuen, S. C., Yang, D., et al. (2019a). Neuroprotective Effects and Hepatorenal Toxicity of Angong Niuhuang Wan against Ischemia-Reperfusion Brain Injury in Rats. Front. Pharmacol. 10, 593. doi:10.3389/fphar.2019.00593

Tsoi, B., Wang, S., Gao, C., Luo, Y., Li, W., Yang, D., et al. (2019b). Realgar and Cinnabar Are Essential Components Contributing to Neuroprotection of Angong Niuhuang Wan with No Hepatorenal Toxicity in Transient Ischemic Brain Injury. Toxicol. Appl. Pharmacol. 377, 114613. doi:10.1016/ j.taap.2019.114613

Vela, L., Gonzalo, O., Naval, J., and Marzo, I. (2013). Direct Interaction of Bax and Bak Proteins with Bcl-2 Homology Domain 3 (BH3)-Only Proteins in Living Cells Revealed by Fluorescence Complementation. J. Biol. Chem. 288, 4935-4946. doi:10.1074/jbc.M112.422204

Wang, G. J., Zhang, L. C., Chen, B., Zhang, Y. Y., Kong, L., and Han, J. (2019). Protective Effects of Salvianolic Acid B on Cerebral Ischemic Reperfusion Injury in Rats. Chin. Arch. Trad Med. 37 (07), 1566-1568. doi:10.13193/ j.issn.1673-7717.2019.07.006

Wang, L., Yuan, Q., Marshall, G., Cui, X., Cheng, L., and Li, Y. (2010). Adverse drug reactions and adverse events of 33 varieties of traditional Chinese medicine injections on National Essential medicines List (2004 edition) of China: an overview on published literatures. J Evid Based Med. 32(2), 95-104. doi:10.1111/j.1756-5391.2010.01073.x

Wang, M., Wang, J., and Liu, Z. (2018). Efects of Intermedin on Autophagy in Cerebral Ischemia/reperfusion Injury. Neuropeptides 68, 15-21. doi:10.1016/ j.npep.2017.10.004

Wang, M., Yao, M., Liu, J., Takagi, N., Yang, B., Zhang, M., et al. (2020a). Ligusticum Chuanxiong Exerts Neuroprotection by Promoting Adult Neurogenesis and Inhibiting Inflammation in the hippocampus of ME Cerebral Ischemia Rats. J. Ethnopharmacol 249, 112385. doi:10.1016/ j.jep.2019.112385

Wang, P., Shao, B. Z., Deng, Z., Chen, S., Yue, Z., and Miao, C. Y. (2018). Autophagy in Ischemic Stroke. Prog. Neurobiol. 163, 98-117. doi:10.1016/ j.pneurobio.2018.01.001

Wang, P. R., Wang, J. S., Zhang, C., Song, X. F., Tian, N., Kong, L. Y., et al. (2013). Decotion Induced Protective Autophagy against the Injury of Cerebral Ischemia/reperfusion via MAPK-mTOR Signaling Pathway. J. Ethnopharmacol 149, 270-280. doi:10.1016/j.jep.2013.06.035

Wang, R., Shi, L., Liu, S., Liu, Z., Song, F., Sun, Z., et al. (2019). Mass SpectrometryBased Urinary Metabolomics for the Investigation on the Mechanism of Action of Eleutherococcus Senticosus (Rupr. \& Maxim.) Maxim. Leaves against Ischemic Stroke in Rats. J. Ethnopharmacol 241, 111969. doi:10.1016/j.jep.2019.111969

Wang, S., Fu, J. L., Hao, H. F., Jiao, Y. N., Li, P. P., and Han, S. Y. (2021). Metabolic Reprogramming by Traditional Chinese Medicine and its Role in Effective Cancer Therapy. Pharmacol. Res. 170, 105728. doi:10.1016/j.phrs.2021.105728

Wang, S. B., Pang, X. B., Zhao, Y., Wang, Y. H., Zhang, L., Yang, X. Y., et al. (2012). Protection of Salvianolic Acid A on Rat Brain from Ischemic Damage via Soluble Epoxide Hydrolase Inhibition. J. Asian Nat. Prod. Res. 14 (11), 1084-1092. doi:10.1080/10286020.2012.723200

Wang, S. F. (2017). The Therapeutic Effect and Mechanism of Mu-Xiang-YouFormulation on Cerebral Ischemia-Reperfusion Injury in Rats. Ningxia, China: Master's Degree of Ningxia Medical University.

Wang, Y., An, R., Umanah, G. K., Park, H., Nambiar, K., Eacker, S. M., et al. (2016). A Nuclease that Mediates Cell Death Induced by DNA Damage and poly(ADPRibose) Polymerase-1. Science 354, 6308. doi:10.1126/science.aad6872

Wang, Y., Kim, N. S., and Haince, J. F. (2011). Poly(ADP-ribose) (PAR) Binding to Apoptosis-Inducing Factor Is Critical for PAR Polymerase-1-dependent Cell Death (Parthanatos). Sci. signaling 4 (167), ra20. doi:10.1126/scisignal.2000902

Wang, Y., Shi, Y., Zou, J., Zhang, X., Wang, M., Guo, D., et al. (2020b). The Intranasal Administration of Carthamus tinctorius L. Extract/phospholipid Complex in the Treatment of Cerebral Infarction via the TNF-K $\beta / M A P K$ Pathway. Biomed. Pharmacother. 130, 110563. doi:10.1016/ j.biopha.2020.110563

Wang, Y., Xiao, G., He, S., Liu, X., Zhu, L., Yang, X., et al. (2020c). Protection against Acute Cerebral Ischemia/reperfusion Injury by QiShenYiQi via Neuroinflammatory Network Mobilization. Biomed. Pharmacother. 125, 109945. doi:10.1016/j.biopha.2020.109945 
Wang, Y. Y. (2016). Internal Medicine of Traditional Chinese Medicine [M]. Shanghai: Shanghai Science and Technology Press, 124-132.

Wang, Z., Yuan, Y., Zhang, Z., and Ding, K. (2019). Inhibition of miRNA-27b Enhances Neurogenesis via AMPK Activation in a Mouse Ischemic Stroke Model. FEBS Open Bio 9, 859-869. doi:10.1002/2211-5463.12614

Wu C. J, C. J., Chen, J. T., Yen, T. L., Jayakumar, T., Chou, D. S., Hsiao, G., et al. (2011). Neuroprotection by the Traditional Chinese Medicine, Tao-Hong-SiWu-Tang, against Middle Cerebral Artery Occlusion-Induced Cerebral Ischemia in Rats. Evid. Based Complement. Alternat Med. 2011, 803015. doi:10.1155/2011/803015

Wu, H. R. (2018). Study on the Regulating Effect of Taohong Siwu Decoction on Brain-Derived Neurotrophic Factor in Rats with Cerebral Ischemia Reperfusion. Hefei, China: Degree. Anhui University of Chinese Medicine.

$\mathrm{Wu}$, J., Zhang, X., and Zhang, B. (2014). Qingkailing Injection for the Treatment of Acute Stroke: a Systematic Review and Meta-Analysis. J. Tradit Chin. Med. 34 (2), 131-139. doi:10.1016/s0254-6272(14)60066-2

Wu, L., Li, F., Zhao, C., Ming, Y., Zheng, C., Li, Y., et al. (2020). Effects and Mechanisms of Traditional Chinese Herbal Medicine in the Treatment of Ischemic Cardiomyopathy. Pharmacol. Res. 151, 104488. doi:10.1016/ j.phrs.2019.104488

Wu, Q. Q., Wang, Y., Senitko, M., Meyer, C., Wigley, W. C., Ferguson, D. A., et al. (2011). Bardoxolone Methyl (BARD) Ameliorates Ischemic AKI and Increases Expression of Protective Genes Nrf2, PPARgamma, and HO-1. Am. J. Physiol. Ren. Physiol 300, F1180-F1192. doi:10.1152/ajprenal.00353.2010

Xiang, Y., Yao, X., Wang, X., Zhao, H., Zou, H., Wang, L., et al. (2019). Houshiheisan Promotes Angiogenesis via HIF-1alpha/VEGF and SDF-1/ CXCR4 Pathways: In Vivo and In Vitro. Biosci. Rep. 39. doi:10.1042/ BSR20191006

Xie, W., Wang, X., Xiao, T., Cao, Y., Wu, Y., Yang, D., et al. (2021). Protective Effects and Network Analysis of Ginsenoside Rb1 against Cerebral Ischemia Injury: A Pharmacological Review. Front. Pharmacol. 2 (12), 604811. doi:10.3389/fphar.2021.604811

Xie, Y., Hou, W., Song, X., Yu, Y., Huang, J., Sun, X., et al. (2016). Ferroptosis: Process and Function. Cell Death Differ 23, 369-379. doi:10.1038/cdd.2015.158

Xu, A. L., Zheng, G. Y., Wang, Z. J., Chen, X. D., and Jiang, Q. (2016). Neuroprotective Effects of Ilexonin A Following Transient Focal Cerebral Ischemia in Rats. Mol. Med. Rep. 13 (4), 2957-2966. doi:10.3892/mmr.2016.4921

$\mathrm{Xu}, \mathrm{L}$. (2019). Research on the Mechanism of Tanshinone IIA Inhibiting Ferroptosis by Regulating Iron Homeostasis in the Model of Cerebral Ischemia. Master Dissertation of Anhui medical university. Anhui Medical University, Hefei. Available at: https://kns.cnki.net/kns8/defaultresult/index.

Xu, Z., Liu, W., and Huang, H. (2020). Astragaloside IV Alleviates Cerebral Ischemia-Reperfusion Injury by Activating the Janus Kinase 2 and Signal Transducer and Activator of Transcription 3 Signaling Pathway. Pharmacology 105 (3-4), 181-189. doi:10.1159/000503361

Yang, B., Sun, Y., Lv, C., Zhang, W., and Chen, Y. (2020). Procyanidins Exhibits Neuroprotective Activities against Cerebral Ischemia Reperfusion Injury by Inhibiting TLR4-NLRP3 Inflammasome Signal Pathway. Psychopharmacology (Berl) 237, 3283-3293. doi:10.1007/s00213-020-05610-z

Yang, C. M., Chen, L. D., and Tao, J. (2012). The Ancient Hinduists in Gualou Guizhi Decoction. Liaoning Zhongyi Zazhi 39 (08), 166-167.

Yang, H., Li, L., Zhou, K., Wang, Y., Guan, T., Chai, C., et al. (2016). Shengmai Injection Attenuates the Cerebral Ischemia/reperfusion Induced Autophagy via Modulation of the AMPK, mTOR and JNK Pathways. Pharm. Biol. 54, 2288-2297. doi:10.3109/13880209.2016.1155625

Yang, Y., and Rosenberg, G. A. (2011). Blood-brain Barrier Breakdown in Acute and Chronic Cerebrovascular Disease. Stroke 42, 3323-3328. doi:10.1161/ STROKEAHA.110.608257

Yao, X., Uchida, K., Papadopoulos, M. C., Zador, Z., Manley, G. T., and Verkman, A. S. (2015). Mildly Reduced Brain Swelling and Improved Neurological Outcome in Aquaporin-4 Knockout Mice Following Controlled Cortical Impact Brain Injury. J. Neurotrauma 32, 1458-1464. doi:10.1089/ neu. 2014.3675

Ye, Y., Li, J., Cao, X., Chen, Y., Ye, C., and Chen, K. (2016). Protective Effect of N-Butyl Alcohol Extracts from Rhizoma Pinelliae Pedatisectae against Cerebral Ischemia-Reperfusion Injury in Rats. J. Ethnopharmacol 188, 259-265. doi:10.1016/j.jep.2016.04.046
Yu, F., Xue, W., Dong, L., Hu, X., Huang, D., and Wang, K. (2019). Tetrahydroxystilbene Glucoside Suppresses NAPDH Oxidative Stress to Mitigate Apoptosis and Autophagy Induced by Cerebral Ischemia/ Reperfusion Injury in Mice. Evid. Based Complement. Alternat Med. 2019, 3913981. doi:10.1155/2019/3913981

Zeng, C., Wang, R., and Tan, H. (2019). Role of Pyroptosis in Cardiovascular Diseases and its Therapeutic Implications. Int. J. Biol. Sci. 15, 1345-1357. doi:10.7150/ijbs. 33568

Zhang, D. S., Fu, M. D., Song, C. L., Wang, C. Y., Lin, X. C., and Liu, Y. L. (2012). Expressions of Apoptosis-Related Proteins in Rats with Focal Cerebral Ischemia after Angong Niuhuang Sticker point Application. Neural Regen. Res. 7 (30), 2347-2353. doi:10.3969/j.issn.1673-5374.2012.30.004

Zhang, D. S., Liu, Y. L., Zhu, D. Q., Huang, X. J., and Luo, C. H. (2015). Point Application with Angong Niuhuang Sticker Protects Hippocampal and Cortical Neurons in Rats with Cerebral Ischemia. Neural Regen. Res. 10 (2), 286-291. doi:10.4103/1673-5374.152384

Zhang, H., Song, Y., and Feng, C. (2020). Improvement of Cerebral Ischemia/ reperfusion Injury by Daucosterol Palmitate-Induced Neuronal Apoptosis Inhibition via PI3K/Akt/mTOR Signaling Pathway. Metab. Brain Dis. 35, 1035-1044. doi:10.1007/s11011-020-00575-6

Zhang, H., Zhai, L., Wang, T., Li, S., and Guo, Y. (2017). Picroside II Exerts a Neuroprotective Effect by Inhibiting the Mitochondria Cytochrome C Signal Pathway Following Ischemia Reperfusion Injury in Rats. J. Mol. Neurosci. 61, 267-278. doi:10.1007/s12031-016-0870-0

Zhang, J., Jiang, Y., Liu, N., Shen, T., Jung, H. W., Liu, J., et al. (2019a). A NetworkBased Method for Mechanistic Investigation and Neuroprotective Effect on Post-treatment of Senkyunolid-H against Cerebral Ischemic Stroke in Mouse. Front. Neurol. 10, 1299. doi:10.3389/fneur.2019.01299

Zhang, J., Liu, M., Huang, M., Chen, M., Zhang, D., Luo, L., et al. (2019b). Ginsenoside F1 Promotes Angiogenesis by Activating the IGF-1/IGF1R Pathway. Pharmacol. Res. 144, 292-305. doi:10.1016/j.phrs.2019.04.021

Zhang J. K, J. K., Yang, L., Meng, G. L., Fan, J., Chen, J. Z., He, Q. Z., et al. (2012). Protective Effect of Tetrahydroxystilbene Glucoside against Hydrogen Peroxide-Induced Dysfunction and Oxidative Stress in Osteoblastic MC3T3E1 Cells. Eur. J. Pharmacol. 689, 31-37. doi:10.1016/j.ejphar.2012.05.045

Zhang, J. Y. (2019). Study on the Mechanism of Resveratrol in Promoting Peripheral Nerve Repair. Doctoral Dissertation of Jilin university. Changchun, China: Jilin university. Available at: https://kns.cnki.net/kns8/ defaultresult/index.

Zhang, K., Li, Y. J., Yang, Q., Gerile, O., Yang, L., Li, X. B., et al. (2013). Neuroprotective Effects of Oxymatrine against Excitotoxicity Partially through Down-Regulation of NR2B-Containing NMDA Receptors. Phytomedicine 20, 343-350. doi:10.1016/j.phymed.2012.10.018

Zhang, Q., Bian, H., Li, Y., Guo, L., Tang, Y., and Zhu, H. (2014). Preconditioning with the Traditional Chinese Medicine Huang-Lian-Jie-Du-Tang Initiates HIF1alpha-dependent Neuroprotection against Cerebral Ischemia in Rats. J. Ethnopharmacol 154, 443-452. doi:10.1016/j.jep.2014.04.022

Zhang Q, Q., Zhao, H., Wang, L., Zhang, Q., and Wang, H. (2012). Effects of WindDispelling Drugs and Deficiency-Nourishing Drugs of Houshiheisan Compound Prescription on Astrocyte Activation and Inflammatory Factor Expression in the Corpus Striatum of Cerebral Ischemia Rats. Neural Regen. Res. 7 (24), 1851-1857. doi:10.3969/j.issn.1673-5374.2012.24.002

Zhang, R., Tang, S., Huang, W., Liu, X., Li, G., Chi, H., et al. (2015). Protection of the Brain Following Cerebral Ischemia through the Attenuation of PARP-1Induced Neurovascular Unit Damage in Rats. Brain Res. 1624, 9-18. doi:10.1016/j.brainres.2015.07.023

Zhang, S., Wang, X., Cheng, F., Ma, C., Fan, S., Xu, W., et al. (2020). Network Pharmacology-Based Approach to Revealing Biological Mechanisms of Qingkailing Injection against Ischemic Stroke: Focusing on Blood-Brain Barrier. Evid. Based Complement. Alternat Med. 2020, 2914579. doi:10.1155/ 2020/2914579

Zhang, S., Zhang, Y., Li, H., Xu, W., Chu, K., Chen, L., et al. (2015). Antioxidant and Anti-excitotoxicity Effect of Gualou Guizhi Decoction on Cerebral Ischemia/reperfusion Injury in Rats. Exp. Ther. Med. 9 (6), 2121-2126. doi:10.3892/etm.2015.2386

Zhang, W., Zhang, Q., Deng, W., Li, Y., Xing, G., Shi, X., et al. (2014). Neuroprotective Effect of Pretreatment with Ganoderma Lucidum in 
Cerebral Ischemia/reperfusion Injury in Rat hippocampus. Neural Regen. Res. 9, 1446-1452. doi:10.4103/1673-5374.139461

Zhang, X., Zhang, Y., Tang, S., Yu, L., Zhao, Y., Ren, Q., et al. (2018). Pien-TzeHuang Protects Cerebral Ischemic Injury by Inhibiting Neuronal Apoptosis in Acute Ischemic Stroke Rats. J. Ethnopharmacol 219, 117-125. doi:10.1016/ j.jep.2018.03.018

Zhang, Y., Fan, L., Li, H., Wang, X., Xu, W., Chu, K., et al. (2018). Gualou Guizhi Granule Protects against Oxidative Injury by Activating Nrf2/ARE Pathway in Rats and PC12 Cells. Neurochem. Res. 43 (5), 1003-1009. doi:10.1007/s11064-018-2507-x

Zhang, Y., Zhang, S., Li, H., Huang, M., Xu, W., Chu, K., et al. (2015). Ameliorative Effects of Gualou Guizhi Decoction on Inflammation in Focal Cerebral Ischemic-Reperfusion Injury. Mol. Med. Rep. 12 (1), 988-994. doi:10.3892/ mmr.2015.3515

Zhao, Q., Cheng, X., Wang, X., Wang, J., Zhu, Y., and Ma, X. (2016). Neuroprotective Effect and Mechanism of Mu-Xiang-You-Fang on Cerebral Ischemia-Reperfusion Injury in Rats. J. Ethnopharmacol 192, 140-147. doi:10.1016/j.jep.2016.07.016

Zhao, T., Tang, H., Xie, L., Zheng, Y., Ma, Z., Sun, Q., et al. (2019). Scutellaria Baicalensis Georgi. (Lamiaceae): a Review of its Traditional Uses, Botany, Phytochemistry, Pharmacology and Toxicology. J. Pharm. Pharmacol. 71 (9), 1353-1369. doi:10.1111/jphp.13129

Zheng, X. W., Shan, C. S., Xu, Q. Q., Wang, Y., Shi, Y. H., Wang, Y., et al. (2018). Buyang Huanwu Decoction Targets SIRT1/VEGF Pathway to Promote Angiogenesis after Cerebral Ischemia/Reperfusion Injury. Front. Neurosci. 12, 911. doi:10.3389/fnins.2018.00911

Zheng, Y. Y., Dong, Z., Lu, X. Q., Xia, Y. P., and Zhu, S. B. (2015). Analysis on 315 Cases of Clinical Adverse Drug Reaction/event Induced by Gastrodin. Zhongguo Zhong Yao Za Zhi 40 (10), 2037-2041.

Zhou, D., Cen, K., Liu, W., Liu, F., Liu, R., Sun, Y., et al. (2021). Xuesaitong Exerts Long-Term Neuroprotection for Stroke Recovery by Inhibiting the ROCKII Pathway, In Vitro and In Vivo. J. Ethnopharmacol 272, 113943. doi:10.1016/ j.jep.2021.113943

Zhou, F., Wang, L., Liu, P., Hu, W., Zhu, X., Shen, H., et al. (2014). Puerarin Protects Brain Tissue against Cerebral Ischemia/reperfusion Injury by Inhibiting the Inflammatory Response. Neural Regen. Res. 9 (23), 2074-2080. doi:10.4103/1673-5374.147934

Zhou, Q. B., Ju, X. N., Wang, X. Y., Wang, M. H., Kong, F., Sun, C., et al. (2016). Pretreatment with Baicalin Attenuates Hypoxia and Glucose DeprivationInduced Injury in SH-Sy5y Cells. Chin. J. Integr. Med. 22 (3), 201-206. doi:10.1007/s11655-015-2326-8

Zhou, X. Y., Luo, Y., Zhu, Y. M., Liu, Z. H., Kent, T. A., Rong, J. G., et al. (2017). Inhibition of Autophagy Blocks Cathepsins-tBid-Mitochondrial Apoptotic Signaling Pathway via Stabilization of Lysosomal Membrane in Ischemic Astrocytes. Cel Death Dis 8, e2618. doi:10.1038/ cddis.2017.34

Zhou, Y., Li, H. Q., Lu, L., Fu, D. L., Liu, A. J., Li, J. H., et al. (2014). Ginsenoside Rg1 Provides Neuroprotection against Blood Brain Barrier Disruption and Neurological Injury in a Rat Model of Cerebral Ischemia/reperfusion through Downregulation of Aquaporin 4 Expression. Phytomedicine 21 (7), 998-1003. doi:10.1016/j.phymed.2013.12.005

Zhou, Z., Dun, L., Wei, B., Gan, Y., Liao, Z., Lin, X., et al. (2020). An H, Musk Ketone Induces Neural Stem Cell Proliferation and Differentiation in Cerebral Ischemia via Activation of the PI3K/Akt Signaling Pathway. Neuroscience 435, 1-9. doi:10.1016/ j.neuroscience.2020.02.031

Zhou, Z. Q., Li, Y. L., Ao, Z. B., Wen, Z. L., Chen, Q. W., Huang, Z. G., et al. (2017). Baicalin Protects Neonatal Rat Brains against Hypoxic-Ischemic Injury by Upregulating Glutamate Transporter 1 via the Phosphoinositide 3-kinase/ protein Kinase B Signaling Pathway. Neural Regen. Res. 12 (10), 1625-1631. doi:10.4103/1673-5374.217335

Zhu, B., Cao, H., Sun, L., Li, B., Guo, L., Duan, J., et al. (2018). Metabolomics-based Mechanisms Exploration of Huang-Lian Jie-Du Decoction on Cerebral Ischemia via UPLC-Q-TOF/MS Analysis on Rat Serum. J. Ethnopharmacol 216, 147-156. doi:10.1016/j.jep.2018.01.015

Zou, H., Long, J., Zhang, Q., Zhao, H., Bian, B., Wang, Y., et al. (2016). Induced Cortical Neurogenesis after Focal Cerebral Ischemia--Three Active Components from Huang-Lian-Jie-Du Decoction. J. Ethnopharmacol 178, 115-124. doi:10.1016/j.jep.2015.12.001

Conflict of Interest: The authors declare that the research was conducted in the absence of any commercial or financial relationships that could be construed as a potential conflict of interest.

Publisher's Note: All claims expressed in this article are solely those of the authors and do not necessarily represent those of their affiliated organizations, or those of the publisher, the editors and the reviewers. Any product that may be evaluated in this article, or claim that may be made by its manufacturer, is not guaranteed or endorsed by the publisher.

Copyright $\odot 2022$ Huang, Wan, Shao, Li, Zhang and He. This is an open-access article distributed under the terms of the Creative Commons Attribution License (CC $B Y)$. The use, distribution or reproduction in other forums is permitted, provided the original author(s) and the copyright owner(s) are credited and that the original publication in this journal is cited, in accordance with accepted academic practice. No use, distribution or reproduction is permitted which does not comply with these terms. 